\title{
A Nonlocal in Time Problem for Evolutionary Singular Equations in Generalized Spaces of Type $\dot{S}$
}

\author{
Hanna Verezhak (iD) and Vasyl Gorodetskyi \\ Faculty of Mathematics and Informatics, Yuriy Fedkovych Chernivtsi National University, 28 Universitetska St., \\ Chernivtsi 58012, Ukraine \\ Correspondence should be addressed to Hanna Verezhak; g.verezhak@gmail.com
}

Received 2 March 2020; Revised 12 May 2020; Accepted 13 May 2020; Published 15 June 2020

Academic Editor: Lars E. Persson

Copyright (C) 2020 Hanna Verezhak and Vasyl Gorodetskyi. This is an open access article distributed under the Creative Commons Attribution License, which permits unrestricted use, distribution, and reproduction in any medium, provided the original work is properly cited.

In this paper, we establish the correct solvability of a nonlocal multipoint in time problem for the evolutionary equation of a parabolic type with the Bessel operator of infinite order in the case where the initial function is an element of the space of generalized functions of type $(S)^{\prime}$.

\section{Introduction}

Singular parabolic equations with the Bessel operator are related to the equations with degeneracy in terms of the spatial variable operator (such equations degenerate on the boundary of domain), and they are close to evenly parabolic equations in terms of their internal properties. They are used in the study of temperature fields, in the construction of mathematical modes of diffusion processes, and in anisotropic media that describe the phenomena of heat and mass transfer, radial oscillations of waves, occur in crystallography, hydrodynamics, and problems of interaction.

The theory of classical solutions of the Cauchy problem for such equations is constructed in the works of M.I. Matiychuk, V.V. Krekhivskyi, S.D. Ivasyshen, V.P. Lavrenchuk, I.I. Verenych, and others. The Cauchy problem for singular parabolic equations in the classes of distributions and ultradistributions was studied by Ya.I. Zhytomyrskyi, V.V. Gorodetskyi, I.V. Zhytariuk, V.P. Lavrenchuk, O.V. Martyniuk, and others.

Gel'fand and Shilov in monograph ([1], p. 203-211) proposed a method of constructing functional spaces of infinitely differentiable functions given on $\mathbb{R}$, which impose certain conditions for decreasing on infinity and increasing derivatives with increasing of order. These conditions are set using inequalities $\left|x^{k} \varphi^{(n)}(x)\right| \leq c_{k n},\{k, n\} \subset$
$\mathbb{Z}_{+}$, where $\left\{c_{k n}\right\}$ is some sequence of positive numbers depending on the function $\varphi$. If sequence $\left\{c_{k n}\right\}$ has a special form then we get a certain subclass of spaces of Schwartz space $S=S(\mathbb{R})$ of rapidly decreasing functions on $\mathbb{R}$. In [1], the case where $c_{k n}=k^{k \alpha} n^{n \beta}, \alpha>0, \beta>0$ are fixed parameters is studied in detail; the corresponding spaces are called spaces of type $S$ and are denoted by the symbol $S_{\alpha}^{\beta}$. Functions from these spaces and all their derivatives decrease on the real axis as $|x| \longrightarrow \infty$ faster than $\exp \{-a$ $\left.|x|^{1 / \alpha}\right\}, a>0, x \in \mathbb{R}$. Such spaces are often used in the study of the problem of the classes of uniqueness and the classes of correct solvability of the Cauchy problem for partial differential equations. In [2-8], it was established that spaces of type $S$ and spaces of the type $S^{\prime}$ that are topologically dual to the spaces $S$ coincide with the sets of initial data for the Cauchy problem in broad classes of partial differential equations of finite and infinite orders for which the solutions are entire functions of the space variables. For example, the fundamental solution of the Cauchy problem for the heat-conduction equation $\partial u / \partial t=\partial^{2} u / \partial x^{2}$ is a function $G(t, x)=(2 \sqrt{\pi t})^{-1}$ $\exp \left\{-x^{2} /(4 t)\right\}$, for every $t>0$, this function is an element of the space $S_{1 / 2}^{1 / 2}$ as a function of $x$ ([7], p. 46) and the space $S_{1 / 2}^{1 / 2}$ of a space of the type $S$.

If $c_{k n}=a_{k} b_{n}$, where $\left\{a_{k}, k \in \mathbb{Z}_{+}\right\},\left\{b_{n}, n \in \mathbb{Z}_{+}\right\}$are some sequences of positive numbers, then we have generalized 
spaces of type $S$, denoted by the symbol $S_{a_{k}}^{b_{n}}$. The spaces $S_{a_{k}}^{b_{n}}$ (their topological structure, properties of functions, and basic operations in such spaces) were studied in [9]. Known spaces of type $W$, introduced by Gurevich [10] (see also ([11], p.717)), in which arbitrary convex functions are used to characterize the behavior of functions at infinity instead of power functions, are also embedded in spaces $S_{a_{k}}^{b_{n}}$ in the particular choice of sequences $\left\{a_{k}\right\}$ and $\left\{b_{n}\right\}$ (see [12]). From the results given in $[9,13]$, it follows that generalized spaces of type $S$ are a natural medium for the study of nonlocal multipoint in time problems for evolutionary pseudodifferential equations (in particular, for equations with operators of differentiation of infinite order), for evolutionary equations with generalized Gelfond-Leontiev differentiation operators of finite and infinite orders.

Spaces consisting of even functions of spaces of type $S$ (in particular, spaces $S_{\alpha}^{\beta}$ ) with the corresponding topology are called spaces of type $S$ and are used in the study of evolutionary singular equations of parabolic type with Bessel operators (see [6], [14]). The purpose of this work is to investigate a nonlocal multipoint time problem for evolutionary singular equations of infinite order in generalized spaces of type $\stackrel{\circ}{S}$.

\section{The Spaces of Test Functions}

Here, we dwell on the spaces $S_{a_{k}}^{b_{n}}$, constructed by the sequences of the form $\left\{b_{n}=n ! \rho_{n}, n \in \mathbb{Z}_{+}\right\},\left\{a_{k}=k ! d_{k}, k \in\right.$ $\left.\mathbb{Z}_{+}\right\}$, where $\left\{\rho_{n}\right\}, \rho_{0}=1$ is the sequence of positive numbers, which has the following properties:

(a) the sequence is monotonically decreasing

(b) $\exists c_{b}>0 \exists \gamma_{1} \in(0,1) \forall n \in \mathbb{N}: \rho_{n-1} / \rho_{n} \leq c_{b} \cdot n^{\gamma_{1}}$

(c) $\lim _{n \rightarrow \infty} \sqrt[n]{\rho_{n}}=0$

(d) $\forall \varepsilon>0 \exists c_{\varepsilon}>0 \forall n \in \mathbb{Z}_{+}: \rho_{n} \geq c_{\varepsilon} \cdot \varepsilon^{n} / n^{n}$

The sequence $\left\{d_{k}, k \in \mathbb{Z}_{+}\right\}, d_{0}=1$, also has properties (a)-(d), with condition (b) having the form: $\exists c_{a}>0$ $\exists \gamma_{2} \in(0,1) \forall n \in \mathbb{N}: d_{k-1} / d_{k} \leq c_{a} \cdot k^{\gamma_{2}}$. An example of a sequence $\left\{\rho_{n}\right\}$ with properties (a-d) can be a sequence $\rho_{n}=(n \beta)^{-n \beta} e^{n \beta}$, where $\beta \in(0,1)$ is a fixed parameter. For example, let us check for the sequence $\rho_{n}$ of property (d). We have that

$$
\begin{aligned}
\rho_{n} & =\frac{e^{n \beta}}{(n \beta)^{n \beta}}=\frac{e^{n \beta}}{(n \beta)^{n \beta}} \cdot \frac{[n(1-\beta)]^{n(1-\beta)}}{[n(1-\beta)]^{n(1-\beta)}} \cdot \frac{e^{n(1-\beta)}}{e^{n(1-\beta)}} \\
& =\frac{e^{n}}{n^{n}} \frac{1}{\left[\beta^{\beta}(1-\beta)^{1-\beta}\right]^{n}} \cdot \frac{[n(1-\beta)]^{n(1-\beta)}}{e^{n(1-\beta)}} \\
& =\frac{e^{n}}{n^{n}} \frac{1}{\omega^{n}} \sup _{\lambda \geq 0} \frac{\lambda^{n}}{\exp \left\{\lambda^{1 /(1-\beta)}\right\}},
\end{aligned}
$$

where $\omega=\beta^{\beta}(1-\beta)^{1-\beta}<1$. If we take arbitrary $\varepsilon>0$ and put $\lambda=\varepsilon$, then we get the inequality $\rho_{n} \geq c_{\varepsilon} \varepsilon^{n} / n^{n}$, where $c_{\varepsilon}=\exp \left\{-\varepsilon^{1 /(1-\beta)}\right\}$. Note that condition (b) for this sequence is satisfied with the parameter $\gamma_{1}=\beta$.

We also consider that the parameters $\gamma_{1}, \gamma_{2}$ in condition (b) for the sequences $\left\{\rho_{n}\right\}$ and $\left\{d_{k}\right\}$ are related by condition (e): $\gamma_{1}+\gamma_{2}=\theta \leq 1$.

By $S_{a_{k}}^{b_{n}}$ we denote a collection of functions $\varphi \in C^{\infty}(\mathbb{R})$, satisfying the condition

$$
\exists c, A, B>0 \forall\{k, n\} \subset \mathbb{Z}_{+} \forall x \in \mathbb{R}:\left|x^{k} \varphi^{(n)}(x)\right| \leq c A^{k} B^{n} a_{k} b_{n} .
$$

$S_{a_{k}}^{b_{n}}$ coincides with the union of the countably-normed spaces $S_{a_{k}, A}^{b_{n}, B}$ by all indices $\{A, B\} \subset \mathbb{N}$, where the symbol $S_{a_{k}, A}^{b_{n}, B}$ denotes a collection of those functions $\varphi \in S_{a_{k}}^{b_{n}}$, that for arbitrary $\delta, \rho>0$ satisfy the inequalities

$$
\left|x^{k} \varphi^{(n)}(x)\right| \leq c_{\delta \rho}(A+\delta)^{k}(B+\rho)^{n} a_{k} b_{n},\{k, n\} \subset \mathbb{Z}_{+}, x \in \mathbb{R} ;
$$

the system of norms in $S_{a_{k}, A}^{b_{n}, B}$ is determined by formulas

$$
\|\varphi\|_{\delta \rho}=\sup _{x, k, n} \frac{\left|x^{k} \varphi^{(n)}(x)\right|}{(A+\delta)^{k}(B+\rho)^{n} a_{k} b_{n}},\{\delta, \rho\} \subset\left\{1, \frac{1}{2}, \frac{1}{3}, \cdots\right\} .
$$

It was established in [9] that the function $\varphi \in C^{\infty}(\mathbb{R})$ belongs to the space $S_{a_{k}}^{b_{n}}$, where $a_{k}=k ! d_{k}, b_{n}=n ! \rho_{n}$, if and only if it extends analytically into the complex plane to the whole function $\varphi(z), z \in \mathbb{C}$, which satisfies the condition:

$$
\exists a, b, c>0 \forall z=x+i y \in \mathbb{C}:|\varphi(z)| \leq c \gamma(a x) \rho(b y),
$$

where

$$
\begin{aligned}
& \gamma(x)= \begin{cases}1, & |x|<1, \\
\inf _{k}\left(a_{k} /|x|^{k}\right), & |x| \geq 1,\end{cases} \\
& \rho(y)= \begin{cases}1, & |y|<1, \\
\sup _{n}\left(|y|^{n} / b_{n}\right), & |y| \geq 1 .\end{cases}
\end{aligned}
$$

We note that $\rho$ is a continuously differentiable, even function in $\mathbb{R}$ that increases monotonically over the interval $[1,+\infty)$. It follows from property $(\mathrm{d})$ (see [9]) that

$$
\exists c_{0}, c>0 \forall y \in \mathbb{R}: \rho(y) \geq c_{0} \exp (c|y|)
$$

For example, if $b_{n}=n^{n \beta}, 0<\beta<1$, then $\rho(y) \sim \exp$ $\left\{|y|^{1 / \beta}\right\}$. In addition, as it is proved in [9], $\ln \rho$ is a convex function on $(0,+\infty)$ in the sense that

$$
\forall\left\{y_{1}, y_{2}\right\} \subset(0,+\infty): \ln \rho\left(y_{1}\right)+\ln \rho\left(y_{2}\right) \leq \ln \rho\left(y_{1}+y_{2}\right) \text {. }
$$


The inequality $\ln \rho\left(y_{1}\right)-\ln \rho\left(y_{1}+y_{2}\right) \leq-\ln \rho\left(y_{2}\right)$ follows from (8).

The function $\rho$ in (6) is related to the sequence $\left\{\rho_{n}\right\}$, which constructs the sequence $\left\{b_{n}=n ! \rho_{n}\right\}$ as follows [9]:

$$
\rho_{n}=\inf _{|\omega| \geq 1}\left(\rho(\omega) /|\omega|^{n}\right)=v_{n}^{-n} \rho\left(v_{n}\right),
$$

where $v_{n}$ is the solution of the equation $\omega \mu(\omega)=n, n \in \mathbb{N}$. The function $\mu(\omega)=\rho^{\prime}(\omega) / \rho(\omega)$ is a nonnegative and continuous function on the interval $(0,+\infty)$; herewith $\ln$ $\rho(\omega)=\int_{0}^{\omega} v(\xi) d \xi, \ln \rho(\omega)=\mu(\tilde{\omega}) \cdot \omega, 0<\tilde{\omega}<\omega$, i.e., $\mu(\tilde{\omega})=$ $\ln \rho(\omega) / \omega$. From properties of the function $\rho$, it follows that $\ln \rho(\omega)$ increases faster than any linear function on the interval $(1 ;+\infty)$, as $\omega \longrightarrow+\infty$; i.e., $\lim _{\omega \rightarrow+\infty} \mu(\omega)=+\infty$. Provided that $\rho^{\prime}(2) / \rho(2)=\mu(2)>1$, we obtain that $\omega \mu(\omega)$ $=n$ possesses a unique solution $v_{n}<n+1, n \in \mathbb{N}$. The sequence of solutions $\left\{v_{n}\right\}$ is increasing and it is unbounded. Indeed, suppose it is not, for example, $\sup _{n \in \mathbb{N}} v_{n}=c<\infty$, then we select a convergent subsequence $v_{n_{k}}, k \in \mathbb{N}$ such that $\lim _{k \rightarrow \infty} v_{n_{k}}=a, a<+\infty$; so we obtain a contradiction, since $v_{n_{k}} \cdot \mu\left(v_{n_{k}}\right)=n_{k}$ and passing to the limit as $k \longrightarrow \infty$ we get $a \cdot \mu(a)=+\infty$.

Since $\gamma(x)=1 / \tilde{\gamma}(x)$, where $\tilde{\gamma}(x)=1,|x|<1$, and $\gamma(x)=$ $\sup \left(|x|^{k} / a_{k}\right)$, if $|x| \geq 1$ then $\gamma$ is a continuously differentiable, even function on $\mathbb{R}$ that monotonically decreases on $[1,+\infty), 0<\gamma(x) \leq 1, x \in \mathbb{R}$. For example, if $a_{k}=k^{k \alpha}, \alpha \in$ $(0,1)$, then the following inequalities are true ([1], p. 204)

$$
\exp \left\{-\frac{\alpha}{e}|x|^{1 / \alpha}\right\} \leq \gamma(x) \leq c \exp \left\{-\frac{\alpha}{e}|x|^{1 / \alpha}\right\}, c=\exp \left\{\frac{\alpha e}{2}\right\}
$$

Function $\ln \gamma$ satisfies on $(0,+\infty)$ the inequality [9].

$$
\ln \gamma\left(x_{1}\right)+\ln \gamma\left(x_{2}\right) \geq \ln \gamma\left(x_{1}+x_{2}\right),\left\{x_{1}, x_{2}\right\} \subset(0,+\infty) .
$$

It follows from the results given in [9], that the sequence $\left\{\varphi_{v}, v \geq 1\right\} \subset S_{a_{k}}^{b_{n}}$ converges to zero in this space if the functions $\varphi_{v}$ and their derivatives of any order converge to zero uniformly on every segment $[a, b] \subset \mathbb{R}$ and satisfy the inequality

$$
\left|x^{k} \varphi_{v}^{(n)}(x)\right| \leq c A^{k} B^{n} a_{k} b_{n},\{k, n\} \subset \mathbb{Z}_{+}, x \in \mathbb{R},
$$

where the constants $c, A, B>0$ are independent of $v$.

A function $g$ is called a multiplicator in the space $S_{a_{k}}^{b_{n}}$, if $g \psi \in S_{a_{k}}^{b_{n}}$ for any function $\psi \in S_{a_{k}}^{b_{n}}$ and the mapping $\psi \longrightarrow$ $g \psi$ is a linear and continuous operator from $S_{a_{k}}^{b_{n}}$ into $S_{a_{k}}^{b_{n}}$. A function $g \in C^{\infty}(\mathbb{R})$ that admits an analytic extension onto the entire complex plane and satisfies the condition [9]:

$$
\forall \varepsilon>0 \exists c_{\varepsilon}>0:|g(z)| \leq c_{\varepsilon}(\gamma(\varepsilon x))^{-1} \rho(\varepsilon y), z=x+i y \in \mathbb{C},
$$

is a multiplicator in the space $S_{a_{k}}^{b_{n}}, a_{k}=k ! d_{k}, b_{n}=n ! \rho_{n}$. The operators of multiplication by $x$, all polynomials, operators of differentiation, shift, and extension are defined and continuous in the spaces $S_{a_{k}}^{b_{n}}, a_{k}=k ! d_{k}, b_{n}=n ! \rho_{n}$, [9].

By $S_{a_{k}}^{b_{n}}$, we denote the collection of all even functions from the space $S_{a_{k}}^{b_{n}}$. Since $S_{a_{k}}^{b_{n}}$ forms a subspace of $S_{a_{k}}^{b_{n}}$, then the topology is naturally introduced in $\hat{S}_{a_{k}}^{b_{n}}$. This space with the corresponding topology is called a main space or a generalized space of type $\dot{S}$, and its elements are called test functions.

By $S_{a_{k}}^{b_{n}}(\mathbb{C})$, we denote the collection of functions that are extensions of functions $\varphi$ from space $S_{a_{k}}^{b_{n}}$ into $\mathbb{C}$. According to the results obtained in [9], the space $S_{a_{k}}^{b_{n}}(\mathbb{C})$ can be represented as a union of the countably-normed spaces $S_{a_{k}, A}^{b_{n}, B}(\mathbb{C})$, where $S_{a_{k}, A}^{b_{n}, B}(\mathbb{C})$ consists of those functions $\varphi \in \mathcal{S}_{a_{k}}^{b_{n}}(\mathbb{C})$ for which inequality $|\varphi(x+i y)| \leq c \gamma(\bar{a} x) \rho(\bar{b} y), z=x+i y \in \mathbb{C}$ is true, where $\bar{a}$ is any positive constant less than $a, \bar{b}$ is any constant greater than $b ; a, b>0$ are constants of inequality (5). If for $\varphi \in \dot{S}_{a_{k}, A}^{b_{n}, B}(\mathbb{C})$, we put

$\|\varphi\|_{p \omega}=\sup _{z \in \mathbb{C}} \frac{|\varphi(z)|}{\gamma(a(1-1 / p) x) \rho((b+\omega) y)}, p \in\{2,3, \cdots\}, \omega \in \mathbb{N}$,

then, these norms are equivalent to the corresponding norms in space $\dot{S}_{a_{k}, A}^{b_{n}, B}$. Therefore, the sequence of functions $\left\{\varphi_{v}(x), v \geq 1\right\} \subset S_{a_{k}}^{b_{n}}, x \in \mathbb{R}$, converges to zero if and only if the sequence of functions $\left\{\varphi_{v}(z), v \geq 1\right\}, z \in \mathbb{C}$, converges to zero uniformly in every bounded domain of the complex plane $\mathbb{C}$, the inequalities

$$
\varphi_{v}(z) \leq c \gamma(a x) \rho(b y), z=x+i y \in \mathbb{C},
$$

are true, where constant $c, A, B>0$ are independent of $v$ [9].

Every integer even function satisfying condition (13) is the multiplicator in the space $\hat{S}_{a_{k}}^{b_{n}}$. An example of a multiplicator in $S_{a_{k}}^{b_{n}}$ is the normalized Bessel function $j_{v}, v>-1 / 2$, which is the solution of the equation $B_{v} u+\lambda u=0$, where $B_{v}$ is Bessel operator; $B_{v}=\left(d^{2} / d x^{2}\right)+(2 v+1 / x)(d / d x)$, $v>-1 / 2$ is fixed parameter, provided that $u(0)=1$, $u^{\prime}(0)=0$. Indeed, the normalized Bessel function is related to the ordinary Bessel function $J_{v}, v>-1 / 2$, of the first kind, so [15]:

$$
j_{v}(x)=\frac{2^{v} \Gamma(v+1)}{x^{v}} J_{v}(x)
$$


It is known (see [15]) that the function $J_{v}$ admits an analytic extension into a complex plane $\mathbb{C}$, and the Poisson integral formula holds

$$
J_{v}(z)=\frac{2}{\sqrt{\pi} \Gamma(v+1 / 2)}\left(\frac{z}{2}\right)^{v} \int_{0}^{\pi / 2} \cos (z \cos t) \sin ^{2 v} t d t .
$$

It follows from relations (16) and (17) that the normalized Bessel function $j_{v}$ of the complex argument $z$ is an integer even function and for $j_{v}$ the integral image is correct:

$$
j_{v}(z)=\frac{2 \Gamma(\nu+1)}{\sqrt{\pi} \Gamma(v+1 / 2)} \int_{0}^{\pi / 2} \cos (z \cos t) \sin ^{2 v} t d t
$$

In view of $\cos z=1 / 2\left(e^{i z}+e^{-i z}\right), z=x+i y \in \mathbb{C}$ and by using (18), we obtain estimate:

$$
\left|j_{v}(z)\right| \leq c_{v} e^{|y|}, z=x+i y \in \mathbb{C}, c_{v}=\sqrt{\pi} \frac{\Gamma(\nu+1)}{\Gamma(v+1 / 2)}, v>-\frac{1}{2} .
$$

Since for any convex functions $\ln \bar{\gamma}(x)$ and $\ln \rho(y)$ and for any $\varepsilon>0$, the inequality

$$
|y| \leq \ln \tilde{\gamma}(\varepsilon x)+\ln \rho(\varepsilon y)+c, c>0,
$$

is true, it follows that

$$
\left|j_{v}(z)\right| \leq c_{v, \varepsilon} e^{\ln \tilde{\gamma}(\varepsilon x)+\ln \rho(\varepsilon y)} \equiv c_{v, \varepsilon}(\gamma(\varepsilon x))^{-1} \rho(\varepsilon y) .
$$

It implies that $j_{v}(x)$ is a multiplicator in space $S_{a_{k}}^{b_{n}}, a_{k}=$ $k ! d_{k}, b_{n}=n ! \rho_{n}$.

According to the results presented in [16], the direct and inverse Bessel transforms

$$
\begin{aligned}
& \psi(\sigma) \equiv F_{B_{v}}[\varphi](\sigma)=\int_{0}^{+\infty} \varphi(x) j_{v}(\sigma x) x^{2 v+1} d x, \sigma \in \mathbb{R}, \\
& \varphi(x) \equiv F_{B_{v}}^{-1}[\psi](x)=\frac{1}{2^{2 v} \Gamma^{2}(\nu+1)} \int_{0}^{+\infty} \psi(\sigma) j_{v}(\sigma x) \sigma^{2 v+1} d \sigma, x \in \mathbb{R}
\end{aligned}
$$

are defined in the spaces $S_{a_{k}}^{b_{n}}$; moreover, if the conditions (a)-(e) are satisfied for the sequences $\left\{\rho_{n}\right\}$ and $\left\{d_{k}\right\}$, then the formula $F_{B_{v}}\left[S_{a_{k}}^{b_{n}}\right]=S_{b_{k}}^{a_{n}}$ is true, moreover, operator $F_{B_{v}}$ is continuous [16]. The spaces $S_{\alpha}^{\beta}$ are partial kind of spaces $S_{a_{k}}^{b_{n}}$. The spaces $S_{\alpha}^{\beta}$ consist of even functions of spaces $S_{\alpha}^{\beta}$ with the same topology; accordingly, the formula $F_{B_{v}}\left[S_{\alpha}^{\beta}\right]=S_{\beta}^{\alpha}$ is correct.
By $T_{x}^{\xi}$, we denote the generalized shift operator corresponding to the Bessel operator [17]:

$$
T_{x}^{\xi} \varphi(x)=b_{v} \int_{0}^{\pi} \varphi\left(\sqrt{x^{2}+\xi^{2}-2 x \xi \cos \omega}\right) \sin ^{2 v} \omega d \omega, \varphi \in{\stackrel{\circ}{b_{n}}}_{a_{k}}
$$

where $b_{v}=\Gamma(v+1) /(\Gamma(1 / 2) \Gamma(v+1 / 2)), v>-1 / 2$. Moreover, as it was proved in [18], the operation of a generalized shift is differentiable (even infinitely differentiable) in the space $S_{a_{k}}^{b_{n}}$.

We define the convolution of two functions of space $S_{a_{k}}^{b_{n}}$ by a formula

$$
(\varphi * \psi)(x)=\int_{0}^{\infty} T_{x}^{\xi} \varphi(x) \psi(\xi) \xi^{2 v+1} d \xi,\{\varphi, \psi\} \subset S_{a_{k}}^{\circ b_{n}}
$$

The formula

$$
F_{B}[\varphi * \psi]=F_{B}[\varphi] \cdot F_{B}[\psi], \forall\{\varphi, \psi\} \subset S_{a_{k}}^{\circ b_{n}}
$$

is true [18].

We note that the operation of multiplication of test functions is defined and continuous in the spaces $S_{a_{k}}^{b_{n}}$.

The spaces $S_{a_{k}}^{b_{n}}$ form topological algebras with respect to the convolution of test functions.

Let us consider the pseudodifferential operator $A_{\varphi}=F_{B}^{-1}$ $\left[\varphi F_{B}\right]$ in the space $S_{a_{k}}^{b_{n}}$. Provided that $\varphi$ is a multiplicator in space $S_{b_{k}}^{a_{n}}$, the operator $A_{\varphi}$ is linear and continuous in space $S_{a_{k}}^{b_{n}}$. It turns out that if we consider the operator $A_{\varphi}$ in space $S_{b_{k}}^{b_{n}}$, then it can be understood as a Bessel operator of "infinite order" in this space (see [18]):

$$
A_{\varphi}=\sum_{k=0}^{\infty} c_{2 k}\left(-B_{v}\right)^{k}, \varphi(\sigma)=\sum_{k=0}^{\infty} c_{2 k} \sigma^{2 k}
$$

\section{The Space of Generalized Functions $\left(\boldsymbol{S}_{a_{k}}^{\circ b_{n}}\right)^{\prime}$}

We denote by $\left(S_{a_{k}}^{b_{n}}\right)^{\prime}$ the space of all linear continuous functionals over the corresponding space of test functions with weak convergence. Linear continuous functionals are called regular generalized functions or regular functionals. The action of such functionals upon the test functions $\varphi \in S_{a_{k}}^{b_{n}}$ is determined by the formula

$$
\langle f, \varphi\rangle=\int_{0}^{\infty} f(x) \varphi(x) x^{2 v+1} d x .
$$

Every locally integrated even function $f$ on $\mathbb{R}$, which satisfies condition

$$
\forall \varepsilon>0 \exists c_{\varepsilon}>0 \forall x \in \mathbb{R}:|f(x)| \leq c_{\mathcal{\varepsilon}}(\gamma(\varepsilon x))^{-1},
$$


generates a regular generalized function $F_{f} \in\left(\dot{S}_{a_{k}}^{b_{n}}\right)^{\prime}:\left\langle F_{f}, \varphi\right\rangle$ $=\int_{0}^{\infty} f(x) \varphi(x) x^{2 v+1} d x, \forall \varphi \in S_{a_{k}}^{b_{n}}$.

The following statement is correct: if locally integrated even functionsfandgon $\mathbb{R}$ satisfying the condition (28) do not coincide on the set of Lebesgue positive measure, then there exists a function $\varphi_{0} \in S_{a_{k}}^{b_{n}}$ such that $\left\langle f, \varphi_{0}\right\rangle \neq\left\langle g, \varphi_{0}\right\rangle$, i.e., $F_{f} \neq F_{g}$. On the contrary, if $F_{f} \neq F_{g}$ then the functionsf andgdo not coincide on set of the Lebesgue positive measure. The proof of this statement is analogous to the proof of the corresponding theorem in [19].

The formulated statement allows us to identify locally integrated functions with the generalized functions $F_{f}$ generated by them from space $\left(\dot{S}_{a_{k}}^{b_{n}}\right)^{\prime}$. It follows from the properties of the Lebesgue integral that the embedding

$$
\stackrel{\circ b_{n}}{S_{a_{k}}} \ni f \longrightarrow F_{f} \in\left(\stackrel{\circ b_{n}}{S_{a_{k}}}\right)^{\prime},
$$

is continuous.

Since the operation of a generalized shift of the argument is defined in space $S_{a_{k}}^{b_{n}}$, we define the convolution of a generalized function $f \in\left(\hat{S}_{a_{k}}^{b_{n}}\right)^{\prime}$ with a test function by the formula

$$
(f * \varphi)(x)=\left\langle f_{\xi}, T_{x}^{\xi} \varphi(x)\right\rangle=\left\langle f_{\xi}, T_{\xi}^{x} \varphi(\xi)\right\rangle,
$$

(the index $\xi$ in $f_{\xi}$ means that the functional $f$ acts upon the test function $T_{\xi}^{x} \varphi(\xi)$ as a function of the argument $\xi$ ).

Let $f \in\left(\dot{S}_{a_{k}}^{b_{n}}\right)^{\prime}$. If $f * \varphi \in \hat{S}_{a_{k}}^{b_{n}}, \forall \varphi \in \dot{S}_{a_{k}}^{b_{n}}$ and the relation $\varphi_{v} \longrightarrow 0$ as $v \longrightarrow+\infty$ in the topology of the space $\hat{S}_{a_{k}}^{b_{n}}$ implies that $f * \varphi_{v} \longrightarrow 0$ as $v \longrightarrow+\infty$ in the topology of the space $\dot{S}_{a_{k}}^{b_{n}}$, then the functional $f$ is called a convolver in the space $S_{a_{k}}^{b_{n}}$.

The Bessel transform of a generalized function $f \in\left(\dot{S}_{a_{k}}^{b_{n}}\right)^{\prime}$ is determined by the relation

$$
\left\langle F_{B}[f], \varphi\right\rangle=\left\langle f, F_{B}[\varphi]\right\rangle, \forall \varphi \in \stackrel{\circ a_{n}}{b_{b_{k}}} .
$$

In view of (31), the properties of the linearity and continuity of the functional $f$ and the properties of the Bessel transform of the test functions, the functional $F_{B}[f]$ is linear and continuous in the space of the test functions $S_{b_{k}}^{a_{n}}$. Thus, the Bessel transform of the generalized function $f$ defined on $S_{a_{k}}^{b_{n}}$ is a generalized function on the space $\hat{S}_{b_{k}}^{a_{n}}$.

If a generalized function $f \in\left(\hat{S}_{a_{k}}^{b_{n}}\right)^{\prime}$ is a convolver in the space $S_{a_{k}}^{b_{n}}$, then for any function $\varphi \in \hat{S}_{a_{k}}^{b_{n}}$, the relation

$$
F_{B}[f * \varphi]=F_{B}[f] F_{B}[\varphi],
$$

is true [18].
The following statement implies the following properties: (1) if the generalized function $f$ is a convolver in space $S_{a_{k}}^{b_{n}}$ then its Bessel transform is a multiplicator in the space $\dot{S}_{b_{k}} a_{k}$; (2) if the generalized function $\mathrm{f}$ is a multiplicator in the space $S_{a_{k}}^{b_{n}}$ then its Bessel transform is a convolver in the space $\delta_{b_{k}}^{a_{n}}$.

\section{A Nonlocal Multipoint in Time Problem}

Let us consider an evolutionary equation

$$
\frac{\partial u}{d t}=A_{\varphi} u(t, x), \quad(t, x) \in(0, T] \times \mathbb{R} \equiv \Pi_{T},
$$

where $A_{\varphi}=F_{B_{\sigma \rightarrow x}}^{-1}\left[\varphi(\sigma) F_{B_{x \rightarrow \sigma}}\right]$ is a pseudodifferential operator in the $S_{b_{k}}^{b_{n}}$, constructed according to a function $\varphi$, which is a multiplicator in the space and such that $e^{\varphi} \in \dot{S}_{b_{k}}^{b_{n}}$. Note that $A_{\varphi}$ can be understood as a Bessel operator of infinite order of appearance $A_{\varphi}=\sum_{k=0}^{\infty} c_{2 k}(-1)^{k} B_{v}^{k}$ (see Section 2). By $P_{b_{k}}^{b_{n}}$, we denote the class of functions (symbols) $\varphi$ satisfying the conditions formulated above. For equation (33) we define a nonlocal multipoint in time problem as follows: to find the solution of equation (33) that satisfies the condition

$$
\left.\mu u(t, \cdot)\right|_{t=0}-\left.\mu_{1} u(t, \cdot)\right|_{t=t_{1}}-\cdots-\left.\mu_{m} u(t, \cdot)\right|_{t=t_{m}}=f
$$

where $m \in \mathbb{N},\left\{\mu, \mu_{1}, \cdots, \mu_{m}\right\} \subset(0,+\infty),\left\{t_{1}, \cdots, t_{m}\right\} \subset(0, T]$, $0<t_{1}<\cdots<t_{m} \leq T$ are fixed numbers and, moreover, $\mu>m$ $\sum_{k=0}^{m} \mu_{k}, f \in S_{b_{k}}^{b_{n}}$. We seek the solution of problem (33), (34) with the help of the Bessel transform specified as follows $u(t, x)=F_{B_{v}}^{-1}[v(t, \sigma)] x,(t, x) \in \Pi_{T}$. For the function $v: \Pi_{T}$ $\longrightarrow \mathbb{R}$, we get the following problem with parameter $\sigma$ :

$$
\frac{d v(t, \sigma)}{d t}=\varphi(\sigma) v(t, \sigma),(t, \sigma) \in \Pi_{T},
$$

$$
\left.\mu \nu(t, \sigma)\right|_{t=0}-\left.\sum_{k=1}^{m} \mu_{k} v(t, \sigma)\right|_{t=t_{k}}=\tilde{f}(\sigma), \sigma \in \mathbb{R},
$$

where $\tilde{f}(\sigma)=F_{B_{v}}[f](\sigma)$. The general solution of equation (35) has the form

$$
v(t, \sigma)=c \exp \{t \varphi(\sigma)\},(t, x) \in \Pi_{T},
$$

where $c=c(\sigma)$ is determined from condition (36). Substituting (37) in (36), we obtain

$$
c=\tilde{f}(\sigma)\left(\mu-\sum_{k=1}^{m} \mu_{k} \exp \left\{t_{k} \varphi(\sigma)\right\}\right)^{-1} .
$$


Then

$v(t, \sigma)=\tilde{f}(\sigma) \exp \{t \varphi(\sigma)\}\left(\mu-\sum_{k=1}^{m} \mu_{k} \exp \left\{t_{k} \varphi(\sigma)\right\}\right)^{-1},(t, x) \in \Pi_{T}$.

Thus, the solution of problem (33), (34) has the form

$$
u(t, x)=c_{v} \int_{0}^{\infty} v(t, \sigma) j_{v}(\sigma x) \sigma^{2 v+1} d \sigma,(t, x) \in \Pi_{T} .
$$

We introduce the notation $G(t, x)=F_{B_{v}}^{-1}[Q(t, \sigma)]$, where

$$
Q(t, \sigma)=\exp \{t \varphi(\sigma)\}\left(\mu-\sum_{k=1}^{m} \mu_{k} \exp \left\{t_{k} \varphi(\sigma)\right\}\right)^{-1} \text {. }
$$

Hence, as a result of formal reasoning, we find

$$
u(t, x)=\int_{0}^{\infty} T_{x}^{\xi} G(t, x) f(\xi) \xi^{2 v+1} d \xi=G(t, x) * f(x),(t, x) \in \Pi_{T} .
$$

Indeed,

$$
u(t, x)=c_{v} \int_{0}^{\infty} Q(t, \sigma)\left(\int_{0}^{\infty} f(\xi) j_{v}(\sigma \xi) \xi^{2 v+1} d \xi\right) j_{v}(\sigma x) \sigma^{2 v+1} d \sigma
$$

Since $j_{v}(\sigma \xi) j_{v}(\sigma x)=T_{x}^{\xi} j_{v}(\sigma x)$, we have

$$
\begin{aligned}
u(t, x)= & \int_{0}^{\infty}\left(c_{v} \int_{0}^{\infty} Q(t, \sigma) T_{x}^{\xi} j_{v}(\sigma \xi) \sigma^{2 v+1} d \sigma\right) \\
& \cdot f(\xi) \xi^{2 v+1} d \xi=\int_{0}^{\infty} T_{x}^{\xi} G(t, x) f(\xi) \xi^{2 v+1} d \xi \\
= & G(t, x) * f(x),(t, x) \in \Pi_{T} .
\end{aligned}
$$

The correctness of these transformations follows from the properties of the function $G$ presented in what follows. The properties of the function $G$ are connected with properties of the function $Q$, because $G=F_{B_{\nu}}^{-1}[Q]$. Thus, first of all, we study the properties of the function $Q(t, \sigma)$ regarded as a function of the argument $\sigma$.

Since $\varphi \in P_{b_{k}}^{b_{n}}$, therefore $e^{\varphi} \in \hat{S}_{b_{k}}^{b_{n}}$. Then (see Section 2) there exist numbers $c_{0}, a, b>0$ such that

$$
\left|e^{\varphi(z)}\right| \leq c_{0} e^{-\ln \tilde{\gamma}(a \sigma)+\ln \rho(b \tau)}, \tilde{\gamma}=1 / \gamma=\rho, z=\sigma+i \tau \in \mathbb{C} .
$$

Further, we assume that the constant $c_{0}>0$ in (45) satisfies the condition: $c_{0} \leq m$, where $m$ is the parameter of the multipoint problem (33), (34). Then

$$
\begin{aligned}
\left|e^{t \varphi(z)}\right| & =\left|e^{\varphi(z)}\right|^{t} \leq\left[c_{0} \exp \{-\ln \tilde{\gamma}(a \sigma)+\ln \rho(b \tau)\}\right]^{t} \\
& \leq c \exp \{-t \ln \tilde{\gamma}(a \sigma)+t \ln \rho(b \tau)\}, c=\max \left\{1, c_{0}^{T}\right\} .
\end{aligned}
$$

Lemma 1. Let $\varphi \in P_{b_{k}}^{b_{n}}$. The following estimates are true for the function

$$
Q(t, s)=\exp \{t \varphi(s)\}, t \in(0, T], s=\sigma+i \tau \in \mathbb{C},
$$

and its derivatives (with respect to the variable s) on $\mathbb{R}$ :

$$
\left|D_{\sigma}^{n} Q_{1}(t, \sigma)\right| \leq \tilde{c} \tilde{b}^{n} n ! \rho_{n} \exp \{-t \ln \tilde{\gamma}(\tilde{a} \sigma)\}, n \in \mathbb{Z}_{+},
$$

where the constants $\tilde{c}, \tilde{a}, \tilde{b}>0$ are independent of $t, \rho_{n}=$ $\inf _{\tau}\left(\rho(\tau) /|\tau|^{n}\right)$. The function $Q(t, s)$ belongs to the space $\hat{S}_{b_{k}}^{b_{n}}(\mathbb{C})$ for a fixed $t \in(0, T]$.

Proof. The inequality

$$
t \ln \tilde{\gamma}(a \sigma) \geq \ln \tilde{\gamma}(t a \sigma),
$$

is true for fixed $t \in(0,1)$. This property follows from the relation

$\ln \tilde{\gamma}(t a \sigma)=\int_{0}^{t a \sigma} \mu(\xi) d \xi=t \int_{0}^{a \sigma} \mu(t y) d y \leq t \int_{0}^{a \sigma} \mu(y) d y=t \ln \tilde{\gamma}(a \sigma)$,

where $\mu(\xi)=\tilde{\gamma}^{\prime}(\xi) / \tilde{\gamma}(\xi)$, and $\mu$ is a nonnegative, continuous function on $\mathbb{R}$, monotone increasing on $[0, \infty)$. Then

$$
\begin{aligned}
-t \ln \tilde{\gamma}(a \sigma) & \leq-\ln \tilde{\gamma}(t a \sigma), \\
e^{-t \ln \tilde{\gamma}(a \sigma)+t \ln \rho(b \tau)} & \leq e^{-\ln \tilde{\gamma}(t a \sigma)+\ln \rho(b \tau)} \\
& =e^{-\ln \tilde{\gamma}\left(a_{1} \sigma\right)+\ln \rho(b \tau)}, a_{1} \\
& =a t, t \in(0,1) .
\end{aligned}
$$

If $t>1$, then the inequality $t \ln \rho(b \tau) \leq \ln \rho(t b \tau)$ is true. Then, $t=[t]+\{t\}$ and

$$
e^{-\ln \tilde{\gamma}(a \sigma)}=e^{-[t] \ln \gamma(a \sigma)} e^{-\{t\} \ln \tilde{\gamma}(a \sigma)} \leq e^{-\{t\} \ln \tilde{\gamma}(a \sigma)} \leq e^{-\ln \tilde{\gamma}\left(a_{2} \sigma\right)},
$$

where $a_{2}=a\{t\}$,

$$
e^{t \ln \rho(b \tau)} \leq e^{\ln \rho(t b \tau)}=e^{\ln \rho\left(b_{1} \tau\right)}, b_{1}=b t .
$$

If $t=n, n \in \mathbb{N}$ then $t=1+n-1$. On this

$$
e^{-\ln \tilde{\gamma}(a \sigma)}=e^{-\ln \gamma(a \sigma)} e^{-(n+1) \ln \tilde{\gamma}(a \sigma)} \leq e^{-\ln \tilde{\gamma}(a \sigma)},
$$

Let $\bar{a}=\min \{a t, a\{t\}\}=a\{t\}$, if $t$ is not integer and $\bar{a}=a$, if $t$ is an integer, $\bar{b}=\max \{b, b T\}$. Then, the inequality

$$
\left|Q_{1}(t, s)\right| \leq c e^{-\ln \tilde{\gamma}(\bar{a} \sigma)+\ln \rho(\bar{b} \tau)},
$$

is true for $t \in(0, T]$. Hence, it follows that $Q_{1}(t, s) \in S_{b_{k}}^{b_{n}}(\mathbb{C})$ for every $t \in(0, T]$. 
In the following considerations, we will use the estimate

$$
\left|Q_{1}(t, s)\right| \leq c e^{-t \ln \tilde{\gamma}(a \sigma)+\ln \rho(\bar{b} \tau)}, s=\sigma+i \tau \in \mathbb{C} .
$$

In view of the integral Cauchy formula, we obtain

$$
D_{\sigma}^{n} Q_{1}(t, \sigma)=\frac{n !}{2 \pi i} \int_{\Gamma_{R}} \frac{Q_{1}(t, s)}{(s-\sigma)^{n+1}} d s, n \in \mathbb{Z}_{+},
$$

where $\Gamma_{R}$ is a circle of radius $R$ centered at a point $\sigma \in \mathbb{R}$. By using (56), we obtain the inequalities

$$
\left|D_{\sigma}^{n} Q_{1}(t, \sigma)\right| \leq \frac{n !}{R^{n}} \max _{s \in \Gamma_{R}}\left|Q_{1}(t, s)\right| \leq \frac{n !}{R^{n}} e^{-t \ln \tilde{\gamma}\left(a \sigma_{0}\right)+\ln \rho(\bar{b} R)},
$$

where $\sigma_{0}$ is a point of maximum of the function exp $\{-t \ln$ $\tilde{\gamma}(a \xi)\}, \xi \in[\sigma-R, \sigma+R]$. Since $\ln \tilde{\gamma}(a \xi)$ is an even function on $\mathbb{R}$ that increases on the interval $[0,+\infty)$, then

$$
\sigma_{0}=\left\{\begin{array}{l}
0, \text { for }|\sigma| \leq R \\
\sigma+R, \text { for } \sigma \leq-R \\
\sigma-R, \text { for } \sigma \geq R
\end{array}\right.
$$

In view the inequality $-\ln \tilde{\gamma}\left(\sigma_{1}+\sigma_{2}\right)+\ln \tilde{\gamma}\left(\sigma_{1}\right) \leq-\ln$ $\tilde{\gamma}\left(\sigma_{2}\right), \sigma_{1}, \sigma_{2}>0$, we prove that there exist constants $\tilde{a}, a_{2}$ $>0, \tilde{a} \leq a$ such that

$$
\begin{aligned}
\forall \sigma & \geq 0, \forall R>0: \exp \left\{-t \ln \tilde{\gamma}\left(a \sigma_{0}\right)\right\} \\
& \leq \exp \left\{-t \ln \tilde{\gamma}(\tilde{a} \sigma) \exp \left\{t \ln \tilde{\gamma}\left(a_{2} R\right)\right\}\right. \\
& \leq \exp \left\{-t \ln \tilde{\gamma}(a \sigma) \exp \left\{\ln \tilde{\gamma}\left(\bar{a}_{2} R\right),\right.\right.
\end{aligned}
$$

where $\bar{a}_{2}=\max \left\{a_{2}, a_{2} T\right\}$. Therefore,

$$
\begin{aligned}
\left|D_{\sigma}^{n} Q_{1}(t, \sigma)\right| \leq & \frac{c n !}{R^{n}} \exp \{-t \ln \tilde{\gamma}(\tilde{a} \sigma)\} \exp \left\{t \ln \tilde{\gamma}\left(a_{2} R\right)\right\} \\
& \exp \{\ln \rho(\bar{b} R)\} \leq \frac{c n !}{R^{n}} \exp \{-t \ln \tilde{\gamma}(\tilde{a} \sigma)\} \\
& \exp \{\ln \rho(\tilde{b} R)\}, \tilde{b}=\bar{b}+\bar{a}_{2} .
\end{aligned}
$$

We used the fact that $\tilde{\gamma}=\rho$, and the inequality of convexity for the function $\ln \rho: \ln \rho(\bar{b} R)+\ln \rho\left(\bar{a}_{2} R\right) \leq \ln$ $\rho\left(\left(\bar{b}+\bar{a}_{2}\right) R\right)$.

For any $n \in \mathbb{Z}_{+}$, the function $g_{n, t}(R)=R^{-n} \exp \{\ln \rho$ $(\tilde{b} R)\}=R^{-n} \rho(\tilde{b} R)$ is differentiable on $(0,+\infty)$ and we obtain the following relations

$$
\lim _{R \rightarrow+\infty} g_{n, t}(R)=+\infty, n \in \mathbb{Z}_{+} ; \lim _{R \rightarrow+0} g_{n, t}(R)= \begin{cases}+\infty, & n \in \mathbb{N}, \\ 1, & n=0 .\end{cases}
$$

from the properties of the function $\rho$. Since $g_{n, t}(R)>0, R \in$ $(0,+\infty)$, this function attains its infimum. Thus,

$$
\begin{aligned}
\left|D_{\sigma}^{n} Q_{1}(t, \sigma)\right| & \leq c n ! \inf _{R} g_{n, t}(R) \exp \{-t \ln \tilde{\gamma}(\tilde{a} \sigma)\} \\
& =c n ! \tilde{b}^{n} \inf \frac{\rho(\tilde{b} R)}{(\tilde{b} R)^{n}} \exp \{-t \ln \tilde{\gamma}(\tilde{a} \sigma)\} \\
& =\tilde{c} \tilde{b}^{n} n ! \rho_{n} \exp \{-t \ln \tilde{\gamma}(\tilde{a} \sigma)\}, \tilde{c}=c .
\end{aligned}
$$

Lemma 1 is proved.

Lemma 2. The function

$$
\begin{aligned}
Q_{2}(\sigma) & =\left(\mu-\sum_{k=1}^{m} \mu_{k} \exp \left\{t_{k} \varphi(\sigma)\right\}\right)^{-1} \\
& =\left(\mu-\sum_{k=1}^{m} \mu_{k} Q_{1}\left(t_{k}, \sigma\right)\right)^{-1}, \sigma \in \mathbb{R},
\end{aligned}
$$

is a multiplicator in the space $S_{b_{k}}^{b_{n}}$.

Proof. In view of (46), the inequalities

$$
Q_{1}\left(t_{k}, \sigma\right) \leq c e^{-t_{k} \ln \tilde{\gamma}(a \sigma)} \leq c, \quad k \in\{1, \cdots, m\},
$$

are true. Since

$$
\mu-\sum_{k=1}^{m} \mu_{k} Q_{1}\left(t_{k}, \sigma\right)=\mu\left(1-\frac{1}{\mu} \sum_{k=1}^{m} \mu_{k} \exp \left\{t_{k} \varphi(\sigma)\right\}\right),
$$

moreover,

$$
\frac{1}{\mu} \sum_{k=1}^{m} \mu_{k} \exp \left\{t_{k} \varphi(\sigma)\right\} \leq \frac{c}{\mu} \sum_{k=1}^{m} \mu_{k}<\frac{m}{\mu} \sum_{k=1}^{m} \mu_{k}<1,
$$

hence, by using the polynomial formula, we get

$$
\begin{aligned}
Q_{2}(\sigma)= & \frac{1}{\mu}\left(1-\frac{1}{\mu} \sum_{k=1}^{m} \mu_{k} Q_{1}\left(t_{k}, \sigma\right)\right)^{-1} \\
= & \frac{1}{\mu} \sum_{r=0}^{\infty} \mu^{-r}\left(\sum_{k=1}^{m} \mu_{k} e^{t_{k} \varphi(\sigma)}\right)^{r}=\sum_{r=0}^{\infty} \mu^{-(r+1)} \\
& \cdot \sum_{r_{1}+\cdots+r_{m}=r} \frac{r !}{r_{1} ! \cdots r_{m} !}\left(\mu_{1} e^{t_{1} \varphi(\sigma)}\right)^{r_{1}} \cdots\left(\mu_{m} e^{t_{m} \varphi(\sigma)}\right)^{r_{m}} \\
= & \sum_{r=0}^{\infty} \mu^{-(r+1)} \sum_{r_{1}+\cdots+r_{m}=r} \frac{r !}{r_{1} ! \cdots r_{m} !} \mu_{1}^{r_{1}} \cdots \mu_{m}^{r_{m}} Q_{1}(\lambda, \sigma),
\end{aligned}
$$


where $\lambda:=t_{1} r_{1}+\cdots+t_{m} r_{m}, Q_{1}(\lambda, \sigma)=e^{\lambda \varphi(\sigma)}$. By using this result and (46), we obtain the inequalities

$$
\begin{aligned}
\left|D_{\sigma}^{n} Q_{2}(\sigma)\right| \leq c \tilde{b}^{n} n ! \rho_{n} \sum_{r=0}^{\infty} \mu^{-(r+1)} & \\
& \quad \sum_{r_{1}+\cdots+r_{m}=r} \frac{r !}{r_{1} ! \cdots r_{m} !} \mu_{0}^{r} \exp \{-\lambda \ln \tilde{\gamma}(a \sigma)\} \\
\leq & c \tilde{b}^{n} n ! \rho_{n} \sum_{r=0}^{\infty} \mu^{-(r+1)} \mu_{0}^{r} \sum_{r_{1}+\cdots+r_{m}=r} \frac{r !}{r_{1} ! \cdots r_{m} !}, n \in \mathbb{N},
\end{aligned}
$$

where $\mu_{0}=\max \left\{\mu_{1}, \cdots \mu_{m}\right\}$. Further, we use the formula

$$
\sum_{r_{1}+\cdots+r_{m}=r} \frac{r !}{r_{1} ! \cdots r_{m} !} m^{r}
$$

Then

$$
\left|D_{\sigma}^{n} Q_{2}(\sigma)\right| \leq c \tilde{b}^{n} n ! \rho_{n} \sum_{r=0}^{\infty} \tilde{\mu}^{r}=c^{\prime} \tilde{b}^{n} n ! \rho_{n}, n \in \mathbb{N}
$$

where $\tilde{\mu}=\mu^{-1} \mu_{0} m<1, c^{\prime}=\mu^{-1} \sum_{r=0}^{\infty} \tilde{\mu}^{r}=\mu^{-1}(1-\tilde{\mu})^{-1}$. By using the last inequality and boundedness of the function $Q_{2}$ on $\mathbb{R}$, we conclude that $Q_{2}$ is a multiplicator in space $S_{b_{k}}^{b_{n}}$. Lemma 2 is proved.

By using relations (56), (71) and the Leibniz formula of differentiation of the product of two functions, we obtain

$$
\begin{aligned}
\left|D_{\sigma}^{n} Q(t, \sigma)\right| & =\left|\sum_{l=0}^{n} C_{n}^{l} D_{\sigma}^{l} Q_{1}(t, \sigma) D_{\sigma}^{n-l} Q_{2}(\sigma)\right| \\
& \leq c c^{\prime} \sum_{l=0}^{n} C_{n}^{l} \tilde{b}^{l} l ! \rho_{l} \tilde{b}^{n-l} \rho_{n-l} e^{-t \ln \tilde{\gamma}(a \sigma)} \\
& \leq c_{1} b_{1}^{n} n ! \rho_{n} \exp \{-t \ln \tilde{\gamma}(a \sigma)\} \\
& \equiv c_{1} b_{1}^{n} b_{n} \exp \{-t \ln \tilde{\gamma}(a \sigma)\}, \sigma \in \mathbb{R},
\end{aligned}
$$

where $c_{1}=c c^{\prime}, b_{1}=2 \tilde{b}, b_{n}=n ! \rho_{n}$, the constants $c_{1}, b>0$ are independent of $t$. By virtue of the last inequality, we conclude that the function $Q(t, \sigma)$ (regarded as a function of $\sigma$ ) is an element of the space $S_{b_{k}}^{b_{n}}$ (for any $t \in(0, T]$ ).

In view of the relation $F_{B_{v}}^{-1}\left[\dot{S}_{b_{k}}^{b_{n}}\right]=S_{b_{k}}^{b_{n}}$, we conclude that $G(t, \cdot)=F_{B_{v}}^{-1}[Q(t, \cdot)] \in S_{b_{k}}^{b_{n}}$ for every $t \in(0, T]$.

In the estimates for the derivatives of the function $G(t, x)$ (with respect to the variable $x$ ), we select the dependence on the parameter $t$.
For this, we note (see [16]) that functions from space $S_{b_{k}}^{b_{n}}$ satisfy the condition

$$
\begin{aligned}
\exists \tilde{c}= & c(\varphi)>0 \exists \tilde{A}=\tilde{A}(\varphi)>0 \exists \tilde{B}=\tilde{B}(\varphi) \\
& >0 \forall x \in[0, \infty) \forall\{k, q\} \subset \mathbb{Z}_{+}:\left|x^{2 k} B_{v}^{q} \varphi(x)\right| \\
& \leq \tilde{c} \tilde{A}^{2 k} \tilde{B}^{2 q} b_{2 k} b_{2 q}, \varphi \in S_{b_{k}} .
\end{aligned}
$$

On the converse, if infinitely differentiable, even function $\varphi$ on $\mathbb{R}$ satisfies condition (73), then (see [16]) $\varphi$ is an element of the space $S_{b_{k}}^{b_{q}}$. In view of this observation, we estimate the function $\sigma^{2 q} B_{v}^{k} G(t, \sigma), \sigma \in \mathbb{R}$, for fixed $\{k, q\} \subset \mathbb{Z}_{+}$. To do this, we use the relation established in [16]:

$$
\sigma^{2 q} B_{v}^{k} F_{b_{v}}[\varphi](\sigma)=\int_{0}^{\infty} B_{v}^{q}\left(x^{2 k} \varphi(x)\right) j_{v}(\sigma x) x^{2 v+1} d x, \forall \varphi \in{\stackrel{\circ}{b_{k}}}_{b_{k}},
$$

from the last relation implies that

$$
\sigma^{2 q} B_{v}^{k} G(t, \sigma)=\int_{0}^{\infty} B_{v}^{q}\left(x^{2 k} Q(t, x)\right) j_{v}(\sigma x) x^{2 v+1} d x .
$$

We note also that for the function $\varphi \in S_{b_{k}}^{b_{q}}$, the following formula is true

$$
B_{v}^{q} \varphi(x)=\sum_{i=0}^{q} c_{i}(v) \frac{\varphi^{(2 q-i)}(x)}{x^{i}}, q \in \mathbb{Z}_{+},
$$

where $c_{i}(v)$ are coefficients dependent on $v$, the functions $\varphi^{(2 q-i)} / x^{i}, i \in\{0,1, \cdots, q\}$, are also elements of space $S_{b_{k}}^{b_{q}}$. Then

$$
\begin{aligned}
& B_{v}^{q}\left(x^{2 k} Q(t, x)\right)=c_{0}(v)\left(x^{2 k} Q(t, x)\right)^{(2 q)} \\
& +c_{1}(v) \frac{\left(x^{2 k} Q(t, x)\right)^{(2 q-1)}}{x}+c_{2}(v) \frac{\left(x^{2 k} Q(t, x)\right)^{(2 q-2)}}{x^{2}} \\
& +\cdots+c_{q}(v) \frac{\left(x^{2 k} Q(t, x)\right)^{(q)}}{x^{q}} .
\end{aligned}
$$

We note that inequality

$$
\begin{aligned}
& \exp \{-t \ln \tilde{\gamma}(a \sigma)\}=\exp \left\{\frac{-t}{2} \ln \tilde{\gamma}(a \sigma)\right\} \\
& \cdot \exp \left\{\frac{-t}{2} \ln \tilde{\gamma}(a \sigma)\right\} \leq \exp \{-t \ln \tilde{\gamma}(\bar{a} \sigma)\} \\
& \cdot \exp \left\{\frac{-t}{2} \ln \tilde{\gamma}(a \sigma)\right\}=\tilde{\gamma}(\bar{a} \sigma) \exp \left\{\frac{-t}{2} \ln \tilde{\gamma}(a \sigma)\right\}
\end{aligned}
$$

is true, where $\bar{a}=a\{t / 2\}$, if $t / 2$ is not integer and $\bar{a}=a$, if $t / 2$ is integer (see the proof of Lemma 1). By using (78) and (72), 
we get that in addition to inequalities (72), the following inequalities are true

$$
\begin{aligned}
\left|x^{2 k} D_{x}^{2 q} Q(t, x)\right| \leq & c_{1} b_{1}^{2 q} b_{2 q} \inf _{k} \frac{b_{2 k}}{|\bar{a} x|^{2 k}}|x|^{2 k} \exp \\
& \cdot\left\{-\frac{t}{2} \ln \tilde{\gamma}(a x)\right\} \leq c_{1} a_{1}^{2 k} b_{1}^{2 q} b_{2 q} b_{2 k} \exp \\
& \cdot\left\{-\frac{t}{2} \ln \tilde{\gamma}(a x)\right\}, a_{1}=\frac{1}{\bar{a}} .
\end{aligned}
$$

In addition,

$$
\begin{aligned}
\exists \tilde{c}_{1}, A_{1}, B_{1} & >0 \forall x \in \mathbb{R} \forall\{l, n\} \subset \mathbb{Z}_{+}\left|x^{2 l-1} D_{x}^{2 n-1} Q(t, x)\right| \\
& =\left|x^{2 l-2}\left(x D_{x}^{2 n-1} Q(t, x)\right)\right| \\
& \leq \tilde{c}_{1}\left(A_{1} a_{1}\right)^{2(l-1)} B_{1}^{2 n} b_{2(l-1)} b_{2 n} \exp \left\{\frac{-t}{2} \ln \tilde{\gamma}(a x)\right\},
\end{aligned}
$$

where $a_{1}=1 / a$ (here taken into account $\left.x D_{x}^{2 n-1} Q(t, x) \in \dot{S}_{b_{k}}^{b_{n}}\right)$ for every $t>0$. According to Leibniz formula of differentiation of the product of two functions

$$
\left(x^{2 k} Q(t, x)\right)^{(2 q)}=\sum_{i=0}^{2 q} C_{2 q}^{i}\left(x^{2 k}\right)^{(i)} Q^{(2 q-i)}(t, x)
$$

Let us present the right part (81) as the sum of two terms

$$
\begin{aligned}
& I_{1}:=\sum_{i=0}^{2 q} C_{2 q}^{2 i}\left(x^{2 k}\right)^{(2 i)} Q^{(2 q-2 i)}(t, x), \\
& I_{2}:=\sum_{i=0}^{2 q} C_{2 q}^{2 i-1}\left(x^{2 k}\right)^{(2 i-1)} Q^{(2 q-2 i+1)}(t, x) .
\end{aligned}
$$

From condition (b) for the sequence $\left\{\rho_{k}\right\}$ (see Section 2) there follows the inequality

$$
\frac{b_{2 k-2}}{b_{2 k}} \leq c_{b} k^{-2\left(1-\gamma_{1}\right)}, c_{b}>0 .
$$

Thus

$$
\begin{aligned}
\frac{b_{2 k-2} b_{2 q-2}}{b_{2 k} b_{2 q}} & \leq c_{b}^{2}\left(\frac{k^{\gamma_{1}} q^{\gamma_{1}}}{k q}\right)^{2} \leq c_{b}^{2}\left(\frac{(\max \{k, q\})^{2 \gamma_{1}}}{k q}\right)^{2} \\
& \leq c_{b}^{2}\left(\frac{(k+q)^{2 \gamma_{1}}}{k q}\right)^{2} \leq \gamma\left(\frac{k+q}{k q}\right)^{2}, \gamma=c_{b}^{2}
\end{aligned}
$$

(here taken into account $2 \gamma_{1} \leq 1$, see Section 2). By using (79) and the last inequality, we get

$$
\begin{aligned}
\left|I_{1}\right| & \leq c_{1} a_{1}^{2 k} b_{1}^{2 q} b_{2 k} b_{2 q}\left(1+\frac{1}{2 !} \prod_{i=0}^{1}(2 k-i)(2 q-i)\right. \\
& \cdot \frac{1}{\left(a_{1} b_{1}\right)^{2}} \frac{b_{2 k-2} b_{2 q-2}}{b_{2 k} b_{2 q}}+\frac{1}{4 !} \prod_{i=0}^{3}(2 k-i)(2 q-i) \frac{1}{\left(a_{1} b_{1}\right)^{4}} \\
& \left.\cdot \frac{b_{2 k-4} b_{2 q-4}}{b_{2 k-2} b_{2 q-2}} \cdot \frac{b_{2 k-2} b_{2 q-2}}{b_{2 k} b_{2 q}}+\cdots\right) \exp \left\{-\frac{t}{2} \ln \tilde{\gamma}(a x)\right\} \\
& \leq c_{1} a_{1}^{2 k} b_{1}^{2 q} b_{2 k} b_{2 q}\left(1+\frac{1}{2 !}\left(\frac{4 \gamma(k+q)}{a_{1} b_{1}}\right)^{2}\right. \\
& \left.+\frac{1}{4 !}\left(\frac{4 \gamma(k+q)}{a_{1} b_{1}}\right)^{4}+\cdots\right) \exp \left\{-\frac{t}{2} \ln \tilde{\gamma}(a x)\right\} \\
& \leq c_{1} a_{1}^{2 k} b_{1}^{2 q} b_{2 k} b_{2 q} \operatorname{ch}\left(\frac{4 \gamma(k+q)}{a_{1} b_{1}}\right) \exp \left\{-\frac{t}{2} \ln \tilde{\gamma}(a x)\right\} .
\end{aligned}
$$

Similarly, by using (80), we have

$$
\begin{aligned}
\left|I_{2}\right| & \leq \tilde{c}_{1}\left(a_{1} A_{1}\right)^{2 k} B_{1}^{2 q} b_{2 k} b_{2 q}\left((2 k)(2 q) \frac{1}{\left(a_{1} A_{1}\right)^{2} B_{1}^{0}} \frac{b_{2 k-2} b_{2 q}}{b_{2 k} b_{2 q}}\right. \\
& +\frac{1}{3 !} \prod_{i=0}^{2}(2 k-i)(2 q-i) \frac{1}{\left(a_{1} A_{1}\right)^{4} B_{1}^{2}} \cdot \frac{b_{2 k-4} b_{2 q-2}}{b_{2 k-2} b_{2 q-2}} \\
& \left.\cdot \frac{b_{2 k-2} b_{2 q-2}}{b_{2 k} b_{2 q}}+\cdots\right) \exp \left\{-\frac{t}{2} \ln \tilde{\gamma}(a x)\right\} \\
& \leq \tilde{c}_{1} \frac{B_{1}}{a_{1} A_{1}}\left(a_{1} A_{1}\right)^{2 k} B_{1}^{2 q} b_{2 k} b_{2 q}\left(\frac{1}{1 !} \frac{4 \gamma(k+q)}{a_{1} A_{1} B_{1}}\right. \\
& \left.+\frac{1}{3 !}\left(\frac{4 \gamma(k+q)}{\left(a_{1} A_{1} B_{1}\right)^{3}}+\cdots\right)\right) \times \exp \left\{-\frac{t}{2} \ln \tilde{\gamma}(a x)\right\} \\
& \leq \frac{\tilde{c}_{1} B_{1}}{a_{1} A_{1}}\left(a_{1} A_{1}^{2 k} B_{1}^{2 q} b_{2 k} b_{2 q} s h\left(\frac{4 \gamma(k+q)}{a_{1} A_{1} B_{1}}\right) \exp \left\{-\frac{t}{2} \ln \tilde{\gamma}(a x)\right\} .\right.
\end{aligned}
$$

This yields

$\left|I_{1}\right|+\left|I_{2}\right| \leq \tilde{c}_{0} c \tilde{B}_{0}^{2 q} b_{2 k} b_{2 q} \exp \left\{\frac{4 \gamma}{d}(k+q)\right\} \exp \left\{-\frac{t}{2} \ln \tilde{\gamma}(a x)\right\} ;$

where $\tilde{c}_{0}=\max \left\{c_{1}, \tilde{c}_{1} B_{1} / a_{1} A_{1}\right\}, \tilde{A}_{0}=\max \left\{a_{1}, a_{1} A_{1}\right\}=a_{1}$. $\max \left\{1, A_{1}\right\}=1 / a \max \left\{1, A_{1}\right\}, \tilde{B}_{0}=\max \left\{B_{1}, B_{2}\right\}, d=\min$ $\left\{a_{1} b_{1}, a_{1} A_{1} B_{1}\right\}=a_{1} \min \left\{b_{1}, A_{1} B_{1}\right\}$.

Since $a_{1}=1 / a$ then $1 / a=\bar{a}\left(\min \left\{b_{1}, A_{1} B_{1}\right\}\right)^{-1} \leq a$ $\left(\min \left\{b_{1}, A_{1} B_{1}\right\}\right)^{-1} \equiv a \cdot \alpha$. Moreover, we can assume that $A$ $\geq 1$, i.e., $\tilde{A}_{0}=(1 / a) A_{1}$. So,

$$
\begin{aligned}
\left|x^{2 k} D_{x}^{2 q} Q(t, x)\right| \leq \tilde{c}\left(\frac{L}{\bar{a}}\right)^{2 k} M^{2 q} b_{2 k} b_{2 q} \exp \\
\cdot\left\{-\frac{t}{2} \ln \tilde{\gamma}(a x)\right\},\{k, q\} \subset \mathbb{Z}_{+},
\end{aligned}
$$


where $L=A_{1} \exp \{4 \gamma \alpha\}, M=\tilde{B}_{0} \exp \{4 \gamma \alpha\}$. The other additions in (77) are evaluated similarly. As a result, we obtain the inequality

$$
\begin{aligned}
\left|B_{v}^{q}\left(x^{2 k} Q(t, x)\right)\right| & \leq c_{2}\left(\frac{A_{2}}{\bar{a}}\right)^{2 k} B_{2}^{2 q} b_{2 k} b_{2 q} \exp \left\{-\frac{t}{2} \ln \tilde{\gamma}(a x)\right\} \\
& \leq c_{2}\left(\frac{A_{2}}{\bar{a}}\right)^{2 k} B_{2}^{2 q} b_{2 k} b_{2 q} \exp \left\{-a_{0} t|x|\right\},
\end{aligned}
$$

where the constants $c_{2}, A_{2}, B_{2}, a_{0}>0$ are independent of $t$. Thus,

$$
\begin{aligned}
\left|\sigma^{2 q} B_{v}^{k} G(t, \sigma)\right| & =\left|\int_{0}^{\infty} B_{v}^{q}\left(x^{2 k} Q(t, x)\right) j_{v}(\sigma x) x^{2 v+1} d x\right| \\
& \leq A_{v} \int_{0}^{\infty}\left|B_{v}^{q}\left(x^{2 k} Q(t, x)\right)\right| x^{2 v+1} d x \mid \\
& \leq c_{3}\left(\frac{A_{2}}{\bar{a}}\right)^{2 k} B_{2}^{2 q} b_{2 k} b_{2 q} \int_{0}^{\infty} x^{2 v+1} e^{-a_{0} t x} d x \\
& =c_{4} t^{-2(v+1)}\left(\frac{A_{2}}{\bar{a}}\right)^{2 k} B_{2}^{2 q} b_{2 k} b_{2 q} ;
\end{aligned}
$$

it takes into account that $\left|j_{v}(\sigma x)\right| \leq A_{v}, A_{v}=\sqrt{\pi} \Gamma(v+1) /$ $\Gamma(\nu+1 / 2),\{\sigma, x\} \subset \mathbb{R}$. Hence

$$
\begin{aligned}
\left|B_{v}^{k} G(t, \sigma)\right| & \leq c_{4}\left(\frac{A_{2}}{\bar{a}}\right)^{2 k} b_{2 k} \cdot t^{-2(v+1)} \inf _{q} \frac{b_{2 q}}{\left(|\sigma| B_{2}^{-1}\right)^{2 q}} \\
& =c_{4} t^{-2(v+1)}\left(\frac{A_{2}}{\bar{a}}\right)^{2 k} b_{2 k} \gamma\left(d_{0} \sigma\right) \\
& =c_{4} t^{-2(v+1)}\left(\frac{A_{2}}{\bar{a}}\right)^{2 k} b_{2 k} \exp \left\{-\ln \tilde{\gamma}\left(d_{0} \sigma\right)\right\},
\end{aligned}
$$

where $d_{0}=B_{2}^{-1}$, the constants $c_{4}, A_{2}, d_{0}>0$ are independent of $t$. Thus, the following statement is true.

Lemma 3. The following inequalities are true for the function $B_{v}^{k} G(t, x), G(t, \cdot) \in \hat{S}_{b_{k}}^{b_{n}}$ for every $t \in(0, T]$ :

$$
\left|B_{v}^{k} G(t, x)\right| \leq L_{0} t^{-2(v+1)}\left(\frac{A_{0}}{\bar{a}}\right)^{2 k} b_{2 k} \exp \left\{-\ln \tilde{\gamma}\left(d_{0} x\right)\right\}, k \in \mathbb{Z}_{+},
$$

where the constants $L_{0}, A_{0}, d_{0}>0$ are independent of $t$.

The function $G(t, x)$ is differentiable with respect to $t$ on the interval $(0, T]$. Indeed, since

$$
G(t, x)=c_{v} \int_{0}^{+\infty} Q(t, \sigma) j_{v}(\sigma x) \sigma^{2 v+1} d \sigma ;
$$

then, formally differentiating (93) under the sign of the integral, we obtain the function $\Lambda(t, \sigma):=\varphi(\sigma) Q(t, \sigma) j_{v}$ $(\sigma x) \sigma^{2 v+1}$. Since $\varphi$ is a multiplicator in the space $\delta_{b_{k}}^{b_{n}}$ then

$$
\forall \varepsilon>0 \exists c_{\varepsilon}>0 \forall \sigma \in \mathbb{R}:|\varphi(\sigma)| \leq c_{\varepsilon} e^{\ln \tilde{\gamma}(\varepsilon \sigma)} .
$$

In addition, by (72), we obtain the estimate

$$
\begin{aligned}
|Q(t, \sigma)| & \leq c e^{-t_{0} \ln \tilde{\gamma}(a \sigma)} \leq c e^{-\ln \tilde{\gamma}(\bar{a} \sigma)}, \sigma \\
& \in[0,+\infty), t \in\left[t_{0}, T\right] \subset(0, T],
\end{aligned}
$$

where $\bar{a}=a\left\{t_{0}\right\}$, if $t_{0}$ is not integer and $\bar{a}=a$, if $t_{0}$ is integer. Hence,

$$
\begin{aligned}
|\Lambda(t, \sigma)| & \leq c e^{\ln \tilde{\gamma}(\varepsilon \sigma)-\ln \tilde{\gamma}(\bar{a} \sigma)} \sigma^{2 v+1}, \sigma \\
& \in[0,+\infty), t \in\left[t_{0}, T\right] \subset(0, T] .
\end{aligned}
$$

It follows from the inequality of the convexity of the function $\ln \tilde{\gamma}$ that

$$
\begin{aligned}
& \ln \tilde{\gamma}(\varepsilon \sigma)-\ln \tilde{\gamma}(\bar{a} \sigma) \\
& \quad \leq-\ln \tilde{\gamma}((\bar{a}-\varepsilon) \sigma)=-\ln \tilde{\gamma}(\overline{\bar{a}} \sigma), \overline{\bar{a}} \\
& \quad=\bar{a}-\varepsilon=\bar{a} / 2>0,
\end{aligned}
$$

for $\varepsilon=\bar{a} / 2$. Since

$$
\exists d>0 \forall \sigma \in[0,+\infty): \exp \left\{-\frac{1}{2} \ln \tilde{\gamma}(\bar{a} \sigma)\right\} \sigma^{2 v+1} \leq d ;
$$

then the integrated function $\exp \{-(t / 2) \ln \tilde{\gamma}(\overline{\bar{a}} \sigma)\}$ is a majorant for $\Lambda(t, \sigma), t \in\left[t_{0}, T\right], \sigma \in[0,+\infty)$. Therefore, the integral of the derivative (with respect to the variable $t$ ) of the integrand in (93) converges uniformly on any interval $\left[t_{0}, T\right] \subset(0, T]$ and therefore the derivative with respect to $t$ under the sign of the integral in (93) can be applied at every point $t \in(0, T]$.

Lemma 4. The function $G(t, \cdot), t \in(0, T]$, regarded as an abstract function of the parameter $t$ with values in the space $\hat{S}_{b_{k}}^{b_{n}}$, is differentiable respect to $t$.

Proof. In view of the continuity of the direct and inverse Bessel transforms in spaces of the type $S$, to prove the lemma, it is sufficient to show that the function $F_{B_{v}}[G(t, \cdot)]=Q(t, \cdot)$, as an abstract function of the parameter $t$ with values in the space $F_{B_{v}}\left[\dot{S}_{b_{k}}^{b_{n}}\right]=\hat{S}_{b_{k}}^{b_{n}}$, is differentiable with respect to $t$. In other words, it is necessary to that the limit relation

$\Phi_{\Delta t}(\sigma):=\frac{1}{\Delta t}[Q(t+\Delta t, \sigma)-Q(t, \sigma)] \longrightarrow \frac{\partial}{\partial t} Q(t, \sigma), \Delta t \longrightarrow 0$,

is true in a sense that:

(1) $D_{\sigma}^{s} \Phi_{\Delta t}(\sigma) \underset{\Delta t \rightarrow 0}{\longrightarrow} D_{\sigma}^{s}(\varphi(\sigma) Q(t, \sigma)), s \in \mathbb{Z}_{+}$, uniformly on every segment $[a, b] \subset \mathbb{R}$ 
(2) $\left|D_{\sigma}^{s} \Phi_{\Delta t}(\sigma)\right| \leq \bar{c} \bar{B}^{s} b_{s} e^{-\ln \tilde{\gamma}(\bar{a} \sigma)}, s \in \mathbb{Z}_{+}$, where the constants $\bar{c}, \bar{a}, \bar{b}>0$ are independent of $\Delta t$ if $\Delta t$ is sufficiently small

The function $Q(t, \sigma),(t, \sigma) \in(0, T] \times \mathbb{R}$, is differentiable with respect to $t$ in the ordinary sense. Hence, by the Lagrange theorem on finite spaces,

$\Phi_{\Delta t}(\sigma)=\varphi(\sigma) Q(t+\theta \Delta t, \sigma), 0<\theta<1, t+\theta \Delta t \leq T$.

Thus,

$$
D_{\sigma}^{s} \Phi_{\Delta t}(\sigma)=\sum_{l=0}^{s} C_{s}^{l} D_{\sigma}^{l} \varphi(\sigma) D_{\sigma}^{s-l} Q(t+\theta \Delta t, \sigma),
$$

and

$$
\begin{aligned}
D_{\sigma}^{s} & \left(\Phi_{\Delta t}(\sigma)-\frac{\partial}{\partial t} Q(t, \sigma)\right) \\
& =\sum_{l=0}^{s} C_{s}^{l} D_{\sigma}^{l} \varphi(\sigma)\left[D_{\sigma}^{s-l} Q(t+\theta \Delta t, \sigma)-D_{\sigma}^{s-l} Q(t, \sigma)\right] .
\end{aligned}
$$

Since

$$
\begin{aligned}
& D_{\sigma}^{s-l} Q(t+\theta \Delta t, \sigma)-D_{\sigma}^{s-l} Q(t, \sigma) \\
& \quad=D_{\sigma}^{s-l+1} Q\left(t+\theta_{1} \Delta t, \sigma\right) \theta \Delta t, 0<\theta_{1}<1,
\end{aligned}
$$

in view of estimates (72), we obtain that

$$
D_{\sigma}^{s-l+1} Q\left(t+\theta_{1} \Delta t, \sigma\right) \theta \Delta t \longrightarrow 0, \Delta t \longrightarrow 0,
$$

uniformly on any segment $[a, b] \subset \mathbb{R}$. Then

$$
D_{\sigma}^{s} \Phi_{\Delta t}(\sigma) \longrightarrow D_{\sigma}^{s}\left(\frac{\partial}{\partial t} Q(t, \sigma)\right),
$$

as $\Delta t \longrightarrow 0$ uniformly on any segment $[a, b] \subset \mathbb{R}$. Thus, condition (1) from relation (99) is satisfied.

Since $\varphi$ is a multiplicator in the space $S_{b_{k}}^{b_{n}}$, then

$$
\begin{aligned}
\forall \varepsilon & >0 \exists c_{\varepsilon}>0 \forall z=\sigma+i \tau \in \mathbb{C}:|\varphi(z)| \\
& \leq c_{\varepsilon} e^{\ln \tilde{\gamma}(\varepsilon \sigma)+\ln \rho(\varepsilon \tau)}, \tilde{\gamma}=1 / \gamma=\rho .
\end{aligned}
$$

Because of the Cauchy integral formula, we have that

$$
\varphi^{(n)}(\sigma)=\frac{n !}{2 \pi i} \int_{\Gamma_{R}} \frac{\varphi(z)}{(z-\sigma)^{n+1}} d z, n \in \mathbb{Z}_{+},
$$

where $\Gamma_{R}$ is a circle of radius $R$ centered at the point $\sigma \in \mathbb{R}$. Then, in view (106), we obtain inequalities

$$
\begin{aligned}
\left|\varphi^{(n)}(\sigma)\right| & \leq \frac{n !}{R^{n}} \max _{z \in \Gamma_{R}}|\varphi(z)| \leq c_{\varepsilon} \frac{n !}{R^{n}} e^{\ln \tilde{\gamma}(\varepsilon(\sigma+R))+\ln \rho(\varepsilon R)} \\
& \leq c_{\varepsilon} n ! \inf _{R} \frac{\rho(\varepsilon R)}{R^{n}} e^{\ln \tilde{\gamma}(\varepsilon(\sigma+R))}, \sigma \geq 0 .
\end{aligned}
$$

For sufficiently large values of $\sigma>0$, the inequality $\varepsilon(\sigma$ $+R) \leq(\varepsilon+R) \sigma$ is true. Since the function $\ln \tilde{\gamma}$ increases monotonically for $\sigma \geq 0$, then at the same values of $\sigma$

$$
\ln \tilde{\gamma}(\varepsilon(\sigma+R)) \leq \ln \tilde{\gamma}((\varepsilon+R) \sigma) .
$$

For all values of $\sigma \geq 0$, the following inequality is true:

$$
\ln \tilde{\gamma}(\varepsilon(\sigma+R)) \leq \ln \tilde{\gamma}((\varepsilon+R) \sigma)+c_{R} .
$$

Therefore, for $\sigma \geq 0$

$$
e^{\ln \tilde{\gamma}(\varepsilon(\sigma+R))} \leq \tilde{c}_{R} e^{\ln \tilde{\gamma}((\varepsilon+R) \sigma)} .
$$

Further, for a given $\varepsilon>0$, we assume that $R=\varepsilon$. Then

$$
\left|\varphi^{(n)}(\sigma)\right| \leq \tilde{c}_{\varepsilon} \varepsilon^{n} n ! \rho_{n} e^{\ln \tilde{\gamma}(2 \varepsilon \sigma)}, n \in \mathbb{Z}_{+} .
$$

By using (112) and estimates for the derivatives of the functions $Q(t, \sigma)$, we find

$$
\begin{aligned}
\left|D_{\sigma}^{s} \Phi_{\Delta t}(\sigma)\right| & \leq \tilde{\tilde{c}}_{\varepsilon} \sum_{l=0}^{s} C_{s}^{l} \varepsilon^{l} b_{l} b_{1}^{s-l} b_{s-l} e^{\ln \tilde{\gamma}(2 \varepsilon \sigma)-(t+\theta \Delta t) \ln \tilde{\gamma}(a \sigma)} \\
& \leq \bar{c} \bar{B}^{s} b_{s} e^{\ln \tilde{\gamma}(2 \varepsilon \sigma)-t \ln \tilde{\gamma}(a \sigma)} \\
& \leq \bar{c} \bar{B}^{s} b_{s} e^{\ln \tilde{\gamma}(2 \varepsilon \sigma)-\ln \tilde{\gamma}(\bar{a} \sigma)}
\end{aligned}
$$

where $\bar{a}=a\{t\}$, if $t$ is not an integer; $\bar{a}=a$, if $t$ is an integer, $\bar{c}=\tilde{\tilde{c}}_{\varepsilon}, \bar{B}=2 \max \left\{\varepsilon, b_{1}\right\}$; and $t+\theta \Delta t>0$. Take $\varepsilon=\bar{a} / 4$. In view of the inequality of convexity for the function $\ln \tilde{\gamma}$, we obtain

$$
\begin{aligned}
& \ln \tilde{\gamma}(2 \varepsilon \sigma)-\ln \tilde{\gamma}(\bar{a} \sigma) \\
& \quad \leq-\ln \tilde{\gamma}((\bar{a}-2 \varepsilon) \sigma) \\
& \quad \equiv-\ln \tilde{\gamma}(\overline{\bar{a}} \sigma), \overline{\bar{a}}=\bar{a}-2 \varepsilon=a / 2>0 .
\end{aligned}
$$

Thus

$$
\left|D_{\sigma}^{s} \Phi_{\Delta t}(\sigma)\right| \leq \bar{c} \bar{B}^{s} b_{s} e^{-\ln \tilde{\gamma}(\bar{a} \sigma)}, \sigma \geq 0
$$

moreover, constants $\bar{c}, \bar{B}, \bar{a}>0$ are independent of $\Delta t$ (for sufficiently small $\Delta t$ ). The case $\sigma<0$ is proved similarly. Lemma is proved.

Corollary 5. The formula

$$
\frac{\partial}{\partial t}(f * G(t, x))=f * \frac{\partial G(t, x)}{\partial t}, \forall f \in\left(\stackrel{\circ b_{n}}{b_{b_{k}}}\right)^{\prime}, t \in(0, T],
$$

is true. 
Proof. By the definition of a convolution of a generalized function with the test function, we find

$$
f * G(t, x)=\left\langle f_{\xi}, T_{x}^{\xi} G(t, \xi)\right\rangle=\left\langle f_{\xi}, T_{\xi}^{x} G(t, \xi)\right\rangle
$$

Then

$$
\begin{aligned}
\frac{\partial}{\partial t}(f * G(t, \cdot)) & =\lim _{\Delta t \rightarrow 0} \frac{1}{\Delta t}[(f * G)(t+\Delta t, x)-(f * G)(t, x)] \\
& =\lim _{\Delta t \rightarrow 0}\left\langle f_{\xi}, \frac{1}{\Delta t}\left[T_{x}^{\xi} G(t+\Delta t, x)-T_{x}^{\xi} G(t, x)\right]\right\rangle .
\end{aligned}
$$

By virtue of Lemma 4, the limit relation

$$
\frac{1}{\Delta t}\left[T_{x}^{\xi} G(t+\Delta t, x)-T_{x}^{\xi} G(t, x)\right] \underset{\Delta t \rightarrow 0}{\longrightarrow} \frac{\partial}{\partial t} T_{x}^{\xi} G(t, x)
$$

is true in a sense of convergence in the topology of the space $S_{b_{k}}^{b_{n}}$, thus

$$
\begin{aligned}
\frac{\partial}{\partial t}(f * G(t, x)) & =\left\langle f_{\xi}, \lim _{\Delta t \rightarrow 0} \frac{1}{\Delta t}\left[T_{x}^{\xi} G(t+\Delta t, x)-T_{x}^{\xi} G(t, x)\right]\right\rangle \\
& =\left\langle f_{\xi}, \frac{\partial}{\partial t} T_{x}^{\xi} G(t, x)\right\rangle=\left\langle f_{\xi}, T_{x}^{\xi} \frac{\partial}{\partial t} G(t, x)\right\rangle \\
& =f * \frac{\partial}{\partial t} G(t, x) .
\end{aligned}
$$

Corollary is proved.

Lemma 6. In the space $S_{b_{k}}^{b_{n}}$, the following limit relation is true

$$
\mu \lim _{t \rightarrow+0} G(t, \cdot)-\sum_{l=1}^{m} \mu_{l} \lim _{t \rightarrow t_{l}} G(t, \cdot)=\delta,
$$

where $\delta$ is the Dirac delta function.

Proof. In view of the continuity of the Fourier transform and the function $G(t, \cdot)$ regarded as an abstract function of the parameter $t$ with values in the space $S_{b_{k}}^{b_{n}}$, we replace the relation (121) by the limit relation

$$
\mu \lim _{t \rightarrow+0} F_{B_{v}}[G(t, \cdot)]-\sum_{l=1}^{m} \mu_{l_{t \rightarrow t_{l}}} \lim _{B_{v}}[G(t, \cdot)]=F_{B_{v}}[\delta]
$$

in the space $\left(S_{b_{k}}^{b_{n}}\right)^{\prime}$. By using the representation of the function $G$, we rewrite relation (122) in the form

$$
\mu \lim _{t \rightarrow+0} Q(t, \cdot)-\sum_{l=1}^{m} \mu_{l_{t \rightarrow t_{l}}} \lim _{(t, \cdot)=1 .}
$$

To prove relation (123), we take an arbitrary function $\psi$ $\in S_{b_{k}}^{b_{n}}$, apply the theorem on the limit transition under the sign of Lebesgue integration, we find

$$
\begin{aligned}
\mu \lim _{t \rightarrow+0}\langle Q(t, \cdot), \psi\rangle-\sum_{l=1}^{m} \mu_{l} \lim _{t \rightarrow t_{l}}\langle Q(t, \cdot), \psi\rangle \\
=\mu \lim _{t \rightarrow+0} \int_{\mathbb{R}} Q(t, \sigma) \psi(\sigma) d \sigma-\sum_{l=1}^{m} \mu_{l} \lim _{t \rightarrow t_{l}} \int_{\mathbb{R}} Q(t, \sigma) \psi(\sigma) d \sigma \\
=\int_{\mathbb{R}}\left[\mu Q(0, \sigma)-\sum_{l=1}^{m} \mu_{l} Q\left(t_{k}, \sigma\right)\right] \psi(\sigma) d \sigma \\
=\int_{\mathbb{R}}\left[\frac{\mu}{\mu-\sum_{k=1}^{m} \mu_{k} Q_{1}\left(t_{k}, \sigma\right)}\right] \psi \\
\left.\quad-\sum_{l=1}^{m} \mu_{l} \frac{Q_{1}\left(t_{l}, \sigma\right)}{\mu-\sum_{k=1}^{m} \mu_{k} Q_{1}\left(t_{k}, \sigma\right)}\right] \psi(\sigma) d \sigma \\
=\int_{\mathbb{R}} \frac{\mu-\sum_{l=1}^{m} \mu_{l} Q_{1}\left(t_{l}, \sigma\right)}{\mu-\sum_{k=1}^{m} \mu_{k} Q_{1}\left(t_{k}, \sigma\right)} \psi(\sigma) d \sigma \\
=\int_{\mathbb{R}} \psi(\sigma) d \sigma=\langle 1, \psi\rangle .
\end{aligned}
$$

This implies that relation (123) is true in the space $\left(S_{b_{k}}^{b_{n}}\right)^{\prime}$. Therefore, the relation (121) holds. Lemma is proved.

By $\left(\dot{S}_{b_{k}, *}^{b_{n}}\right)^{\prime}$, we denote a class of generalized functions from $\left(S_{b_{k}}^{b_{n}}\right)^{\prime}$ that are convolvers in the space $S_{b_{k}}^{b_{n}}$.

Corollary 7. Let $\omega(t, x)=f * G(t, x), f \in\left(S_{b_{k} *}^{b_{n}}\right)^{\prime},(t, x) \in$ $\Pi_{T}$. Then, the relation

$$
\mu \lim _{t \rightarrow+0} \omega(t, \cdot)-\sum_{k=1}^{m} \mu_{k_{t \rightarrow t_{k}}} \lim _{t} \omega(t, \cdot)=f
$$

is true in the space $\left(S_{b_{k}}^{b_{n}}\right)^{\prime}$.

Proof. Since $f$ is convolver in the space $S_{b_{k}}^{b_{n}}$, we obtain

$$
F_{B_{v}}[f * G(t, \cdot)]=F_{B_{v}}[f] \cdot F_{B_{v}}[G(t, \cdot)]=F_{B_{v}}[f] \cdot Q(t, \cdot) .
$$

$\left(F_{B_{v}}[f]\right.$ is multiplicator in the space $\left.S_{b_{k}}^{b_{n}}\right)$. By using this fact and the property of the continuity of the Bessel transform, we write (125) in the form 


$$
\begin{aligned}
& \mu \lim _{t \rightarrow+0} F_{B_{v}}[\omega(t, \cdot)]-\sum_{k=1}^{m} \mu_{k_{t \rightarrow t_{k}}} \operatorname{Fim}_{B_{v}}[\omega(t, \cdot)] \\
& =F_{B_{v}}[f]\left(\mu \lim _{t \rightarrow+0} Q(t, \cdot)-\sum_{k=1}^{m} \mu_{k} \lim _{t \rightarrow t_{k}} Q(t, \cdot)\right)=F_{B_{v}}[f],
\end{aligned}
$$

(the relation is considered in space $\left(\hat{S}_{b_{k}}^{b_{n}}\right)^{\prime}$ ). In view (123), we obtain (125). The statement is proved.

The function $\omega(t, \cdot)$ is a solution of equation (33). Indeed $f$ is convolver in the space $S_{b_{k}}^{b_{n}}$, we get

$$
\begin{aligned}
A_{\varphi} u(t, x) & =F_{B_{v}}^{-1}\left[\varphi(\sigma) F_{B_{v}}[f * G(t, \cdot)]\right] \\
& =F_{B_{v}}^{-1}\left[\varphi(\sigma) F_{B_{v}}[f] Q(t, \sigma)\right] \\
& =F_{B_{v}}^{-1}\left[\frac{\partial}{\partial t} Q(t, \sigma) F_{B_{v}}[f]\right] \\
& =F_{B_{v}}^{-1}\left[F_{B_{v}}\left[\frac{\partial}{\partial t} G(t, \cdot)\right] \cdot F_{B_{v}}[f]\right] \\
& =F_{B_{v}}^{-1}\left[F_{B_{v}}\left[f * \frac{\partial G(t, \cdot)}{\partial t}\right]\right]=f * \frac{\partial G(t, \cdot)}{\partial t} .
\end{aligned}
$$

On the other hand (see Corollary 5)

$$
\frac{\partial}{\partial t}(f * G(t, \cdot))=f * \frac{\partial G(t, \cdot)}{\partial t} .
$$

This implies that the function $\omega(t, \cdot)$ satisfies equation (33) in the ordinary sense. By Corollary 7, the nonlocal $m$-point in time problem for equation (33) can be formulated as follows: to find a solution of equation (33) satisfying the condition

$$
\mu \lim _{t \rightarrow+0} u(t, \cdot)-\sum_{k=1}^{m} \mu_{k_{t \rightarrow t_{k}}} \lim _{t}(t, \cdot)=f, f \in\left(\stackrel{\circ b_{n}}{S_{b_{k *}}}\right)^{\prime},
$$

where the limit relation (130) is considered in space $\left(\hat{S}_{b_{k}}^{b_{n}}\right)^{\prime}$ (the restriction imposed on the parameters $\mu, \mu_{1}, \cdots, t_{1}$, $\cdots$ are the same as in problem (33), (34)).

It follows from the above that the function $u(t, x)=f *$ $G(t, x), f \in\left(S_{b_{k *}}^{b_{n}}\right)^{\prime}$, is a solution of (33). If $f=\delta \in\left(\hat{S}_{b_{k *}}^{b_{n}}\right)^{\prime}$, then $f * G(t, x)=G(t, x)$ i.e., $G(t, \cdot)$ is also a solution of (33). By using this fact and relation (121), in what follows the function $G$ is called the fundamental solution of problem (33), (130).

Theorem 8. The problem (33), (130) is correctly solvable. Its solution is given by the formula $u(t, x)=f * G(t, x),(t, x) \in$ $\Pi_{T}, u(t, \cdot) \in S_{b_{k}}^{b_{n}}$ for every $t \in(0, T]$.

Proof. The function $f * G(t, x)$ satisfies (33). The solution $u(t, x)$ continuously depends on the function $f$ in condition (130) in the sense that if $\left\{f, f_{n}, n \geq 1\right\} \subset\left(S_{b_{k *}}^{b_{n}}\right)^{\prime}$ and $f_{n} \longrightarrow f$ as $n \longrightarrow \infty$ in the space $\left(\dot{S}_{b_{k}}^{b_{n}}\right)^{\prime}$, then $u_{n}(t, \cdot)=$ $f_{n} * G(t, \cdot) \longrightarrow u(t, \cdot)=f * G(t, \cdot), n \longrightarrow \infty$ in the space $\left(\hat{S}_{b_{k}}^{b_{n}}\right)^{\prime}$. This property follows from the property of continuity of convolution.

It remains to show that problem (33), (130) possesses a unique solution. To this end, we consider the Cauchy problem

$$
\begin{aligned}
& \frac{\partial v}{\partial t}+A_{\varphi}^{*} v=0,(t, x) \in\left[0, t_{0}\right) \times \mathbb{R}, 0 \leq t<t_{0} \leq T, \\
& \left.v(t, \cdot)\right|_{t=t_{0}}=g, g \in\left(\stackrel{\circ b_{n}}{S_{b_{k *}}}\right),
\end{aligned}
$$

where $A_{\varphi}^{*}$ is the restriction of operator adjoint to the operator $A$ to the space $\dot{S}_{b_{k}}^{b_{n}} \subset\left(\dot{S}_{b_{k}}^{b_{n}}\right)^{\prime}$. We understand condition (132) in a weak sense. The Cauchy problem (131), (132) is solvable, moreover $v(t, \cdot) \in \hat{S}_{b_{k}}^{b_{n}}$ for every $t \in\left[0, t_{0}\right)$.

Let $Q_{t_{0}}^{t}:\left(\hat{S}_{b_{k *}}^{b_{n}}\right)^{\prime} \longrightarrow S_{b_{k}}^{b_{n}}$ be an operator that associates a functional $g \in\left(\hat{S}_{b_{k *} b_{n}}\right)^{\prime}$ with a solution of problem (131), (132). The operator $Q_{t_{0}}^{t}$ is linear and continuous, it is defined for any $t$ and $t_{0}$ such that $0 \leq t<t_{0} \leq T$ and has the properties

$$
\forall g \in\left(\begin{array}{l}
{ }^{\circ} b_{n} \\
S_{b_{k *}}
\end{array}\right),: \frac{d Q_{t_{0}}^{t} g}{d t}+A_{\varphi}^{*} Q_{t_{0}}^{t} g=0, \lim _{t \rightarrow t_{0}} Q_{t_{0}}^{t} g=g
$$

(the limit is considered in the space $\left.\left(\hat{S}_{b_{k}}^{b_{n}}\right)^{\prime}\right)$.

Let us consider a solution $u(t, x),(t, x) \in \Pi_{T}$ of problem (33), (130) understood as a regular functional from the space $\left(\hat{S}_{b_{k *}}^{b_{n}}\right)^{\prime} \supset \hat{S}_{b_{k}}^{b_{n}}$. We prove that problem (33), (130) may have only one solution in space $\left(\dot{S}_{b_{k *}}^{b_{n}}\right)^{\prime}$. To this end, it suffices to prove that only the functional $u(t, x) \equiv 0$ (for any $t \in(0, T]$ ) can be a unique solution of Eq. (33) with the trivial boundary condition (130). We apply the functional $u$ to a function $Q_{t_{0}}^{t} g \in \hat{S}_{b_{k}}^{b_{n}}$, where $g$ is an arbitrarily fixed element from the space $S_{b_{k}}^{b_{n}} \subset\left(S_{b_{k *}}^{b_{n}}\right)^{\prime}$. Further, differentiating with respect to $t$ and using Eq. (33), (131) we get

$$
\begin{aligned}
\frac{\partial}{\partial t}\left\langle u, Q_{t_{0}}^{t} g\right\rangle & =\left\langle\frac{\partial u}{\partial t}, Q_{t_{0}}^{t} g\right\rangle+\left\langle u, \frac{\partial Q_{t_{0}}^{t} g}{\partial t}\right\rangle \\
& =\left\langle A_{\varphi} u, Q_{t_{0}}^{t} g\right\rangle-\left\langle u, A_{\varphi}^{*} Q_{t_{0}}^{t} g\right\rangle \\
& =\left\langle A_{\varphi} u, Q_{t_{0}}^{t} g\right\rangle-\left\langle A_{\varphi} u, Q_{t_{0}}^{t} g\right\rangle \\
& =0, t \in\left[0, t_{0}\right) .
\end{aligned}
$$

This implies that $\left\langle u(t, \cdot), Q_{t_{0}}^{t} g\right\rangle$ is constant. By using properties of abstract functions, we obtain the relation

$$
\lim _{t \rightarrow t_{0}}\left\langle u(t, \cdot), Q_{t_{0}}^{t} g\right\rangle=\left\langle u\left(t_{0}, \cdot\right), g\right\rangle=\text { const } \equiv c,
$$


at any point $t_{0} \in(0, T]$.Thus, if $f=0$ in (130) then

$$
\mu \lim _{t \rightarrow+0}\langle u(t, \cdot), g\rangle-\sum_{k=1}^{m} \mu_{k_{t \rightarrow t_{k}}}\langle u(t, \cdot), g\rangle=\mu c_{0}-\sum_{k=1}^{m} \mu_{k} c_{k}=0 .
$$

This implies that $c_{0}=c_{1}=\cdots=c_{m}=0$. Suppose it is not. For example, $c_{0} \neq 0$. Then we get a relation $\mu-\sum_{k=1}^{m} \mu_{k} \alpha_{k}=$ 0 , where $\alpha_{k}=c_{k} / c_{0}$, i.e., $\mu=\sum_{k=1}^{m} \mu_{k} \alpha_{k}$. Since $\alpha_{k}$ is any constant, and by $\mu, \mu_{1}, \cdots, \mu_{m}$ are fixed parameters, and $\mu>$ $\sum_{k=1}^{m} \mu_{k}$, the obtained contradiction proves that $c_{0}=0$. Similarly, we prove that $c_{1}=c_{2}=\cdots=c_{m}=0$. Hence, $\left\langle u\left(t_{0}, \cdot\right), g\right\rangle$ $=0$ for any $g \in S_{b_{k}}^{b_{n}}$, i.e., $u\left(t_{0}, x\right)$ is a zero functional from the space $\left(S_{b_{k *}}^{b_{n}}\right)^{\prime}$. Since $t_{0} \in(0, T]$ and $t_{0}$ is arbitrary then $u$ $(t, x)=0$ for all $t \in(0, T]$. Theorem is proved.

As an example, we consider equation (33) with the operator $A_{\varphi}$, constructed on the basic of the function $\varphi(x)=-x^{2}$, $x \in \mathbb{R}$. In this case, we obtain

$$
A_{\varphi}=B_{v}=\frac{d^{2} u}{d x^{2}}+(2 v+1)^{-1} x^{-1} \frac{d}{d x}, v>-1 / 2
$$

and equation (33) is the equation with the Bessel operator

$$
\frac{\partial u}{\partial t}=\frac{\partial^{2} u}{\partial x^{2}}+\frac{2 v+1}{x} \frac{\partial u}{\partial x}, v>-\frac{1}{2},(t, x) \in \Pi_{T}
$$

The function $\varphi(x)=-x^{2}$ is an element of the space $P_{1 / 2}^{1 / 2}$ $\equiv P_{k^{k / 2}}^{n^{n / 2}} \equiv P_{b_{k}}^{b^{n}}$. Indeed, $e^{-x^{2}} \in S_{1 / 2}^{1 / 2}$, because

$$
\left|e^{-z^{2}}\right|=\left|e^{-(x+i y)^{2}}\right|=e^{-x^{2}+y^{2}}, z=x+i y \in \mathbb{C}
$$

From the characteristic of the spaces $S_{\alpha}^{\beta}$ and (139) imply that $e^{-x^{2}} \in S_{\alpha}^{\beta}$, where $\alpha=1 / 2,1 / 1-\beta=2$, i.e., $\beta=1 / 2$. In addition, the function $-x^{2}, x \in \mathbb{R}$ is a multiplicator in the space $S_{1 / 2}^{1 / 2}$. In this case, the constant $c_{0}$ in inequality (45) is equal to one, i.e., the condition $c_{0} \leq m, m \in \mathbb{N}$ is satisfied. By the above theorem, the nonlocal $m$-point in time problem for equation (138) is correctly solvable if $f \in\left(S_{1 / 2}^{1 / 2},\right)^{\prime}$, herewith $u(t, x)=f * G(t, x)$, where

$$
\begin{aligned}
G(t, x)= & 2^{-v} \Gamma^{-1}(v+1) \sum_{r=0}^{\infty} \frac{1}{\mu^{(r+1)}} \sum_{r_{1}+\cdots+r_{m}=r} \\
& \cdot \frac{r ! \mu_{1}^{r_{1}} \cdots \mu_{m}^{r_{m}}}{r_{1} ! \cdots r_{m} !}(2 \lambda(t, r))^{-(v+1)} \\
& \times \exp \left\{-x^{2} /(4 \lambda(t, r))\right\},
\end{aligned}
$$

where $\lambda(t, r)=t_{1} r_{1}+\cdots+t_{m} r_{m}+t$. In particular, if $f=\delta \epsilon$ $\left(S_{1 / 2}^{1 / 2},{ }^{\prime}\right)^{\prime}, m=1, t_{1}=T$ (the case of two-point problem), then

$$
\begin{aligned}
u(t, x)= & G(t, x)=2^{-(2 v+1)} \mu^{-1} \Gamma^{-1}(\nu+1) \sum_{r=0}^{\infty}\left(\frac{\mu_{1}}{\mu}\right)^{r} \\
& \cdot \frac{1}{(t+r T)^{v+1}} \exp \left\{\frac{-x^{2}}{4(t+r T)}\right\} .
\end{aligned}
$$

\section{Conclusion}

The correct solvability of a nonlocal multipoint in time problem for the evolutionary equation of a parabolic type with the Bessel operator of infinite order of appearance $\sum_{k=0}^{\infty} c_{2 k}\left(-B_{0}\right)^{k}$ in the case where the initial function is an element of the space of generalized functions of type $\left(S^{\prime}\right)^{\prime}$ is established in this paper. The properties of the fundamental solution this problem are investigated.

\section{Data Availability}

No data were used to support this study.

\section{Conflicts of Interest}

The authors declare that there is no conflict of interests regarding the publication of this article.

\section{Acknowledgments}

This study was conducted in the framework of postgraduate study in Yuriy Fedkovych Chernivtsi National University.

\section{References}

[1] I. M. Gel'fand and G. E. Shilov, Spaces of Test and Generalized Functions [in Russian], Fizmatgiz, Moscow, 1958.

[2] V. I. Gorbachuk and M. L. Gorbachuk, Boundary-Value Problems for Differential-Operator Equations [in Russian], Naukova Dumka, Kyiv, 1984.

[3] M. L. Gorbachuk and V. I. Gorbachuk, Boundary-Value Problems for Operator Differential Equations, Kluwer, Dordrecht, 1991.

[4] A. I. Kashpirovskii, Boundary Values of Solutions for some Classes of Homogeneous Differential Equations in Hilbert Spaces [in Russian], Candidate-Degree Thesis (Physics and Mathematics), Kyiv, 1981.

[5] M. L. Gorbachuk and P. I. Dudnikov, "On the initial data of the Cauchy problem for parabolic equations for which solutions are infinitely differentiable," Dopovidi Akademii Nauk Ukrainskoi RSR. Seriya A, no. 4, pp. 9-11, 1981.

[6] V. V. Horodets'kyi, Limit Properties of the Solutions of Equations of Parabolic Type Smooth in a Layer [in Ukrainian], Ruta, Chernivtsi, 1998.

[7] V. V. Horodets'kyi, Sets of Initial Values of Smooth Solutions of Differential-Operator Equations of Parabolic Type [in Ukrainian], Ruta, Chernivtsi, 1998.

[8] V. V. Horodets'kyi, Evolutionary Equations in Countably Normed Spaces of Infinitely Differentiable Functions [in Ukrainian], Ruta, Chernivtsi, 2008.

[9] V. V. Gorodetskii and O. V. Martynyuk, “Gel'fand-Leont'ev operators of generalized differentiation in spaces of the type 
S," Sibirskii Matematicheskii Zhurnal, vol. 54, no. 3, pp. 569584, 2013.

[10] B. L. Gurevich, "Some spaces of test and generalized functions and the Cauchy problem for finite-difference schemes," Doklady Akademii Nauk SSSR, vol. 99, no. 6, pp. 893-896, 1954.

[11] I. M. Gel'fand and G. E. Shilov, Some Problems of the Theory of Differential Equations [in Russian], Fizmatgiz, Moscow, 1958.

[12] T. I. Hotychan and R. M. Atamanyuk, "Various ways of definition of spaces of the type W," Naukovyi Visnyk Chernivetskogo Universytetu. Matematyka, vol. 111, no. 111, pp. 21-26, 2001.

[13] V. V. Gorodetsky, R. I. Petryshyn, and T. S. Todoriko, "Nonlocal time multipoint nonlocal problem for a certain class of infinite order partial differential equations," Nonlinear Oscillations, vol. 18, no. 2, pp. 176-191, 2015.

[14] Y. I. Zhytomyrskiy, "The Cauchy problem for systems of linear partial differential equations with a differential Bessel operator," Matematicheskii Sbornik, vol. 36, no. 2, pp. 299-310, 1955.

[15] T. Korn and G. Korn, Handbook of Mathematics [in Russian], Science, Moscow, 1977.

[16] V. V. Gorodetskyi and T. I. Gotinchan, "Transformation of Bessel in spaces of type $S$," Bukovinian Mathematical Journal, vol. 5, no. 3-4, pp. 50-55, 2017.

[17] B. M. Levitan, "Expansion in Fourier series and integrals with Bessel functions," Uspekhi Matematicheskikh Nauk, vol. 6, no. 2, pp. 102-143, 1951.

[18] V. V. Gorodetskyi and G. P. Verezhak, "Generalized of $S$ type spaces," Bukovinian Mathematical Journal, vol. 5, no. 1-2, pp. 49-61, 2017.

[19] I. M. Gel'fand and G. E. Shilov, "Fourier transforms of rapidly increasing functions and questions of uniqueness of the solution of Cauchy's problem," Uspekhi Matematicheskikh Nauk, vol. 8, no. 6, pp. 3-54, 1953. 